\title{
The whole profiling and competing endogenous RNA network analyses of noncoding RNAs in adipose stem cells from diabetic, old and young patients
}

\section{Sen Ren \\ Wuhan Union Hospital \\ Hewei xiong \\ Wuhan Union Hospital \\ Jing Chen \\ Wuhan Union Hospital \\ Xiaofan Yang \\ Wuhan Union Hospital \\ Yutian Liu \\ Wuhan Union Hospital \\ Jiahe Guo \\ Wuhan Union Hospital \\ Tao Jiang \\ Wuhan Union Hospital \\ Zhao Xu \\ Wuhan Union Hospital \\ Meng Yuan \\ Wuhan Union Hospital \\ Yang Liu \\ Wuhan Union Hospital \\ Nan Zhou \\ Wuhan Union Hospital \\ Hongrui Chen \\ Wuhan Union Hospital \\ Wenqing $\mathrm{Li}$ \\ Wuhan Union Hospital \\ Hans-Günther Machens}

Technical University of Munich: Technische Universitat Munchen

Zhenbing Chen ( $\nabla$ zbchen@hust.edu.cn ) 
Union Hospital of Tongji Medical College of Huazhong University of Science and Technology https://orcid.org/0000-0003-2828-866X

\section{Research}

Keywords: adipose stem cell, noncoding RNAs, Competing endogenous RNA, diabetes, aging

Posted Date: January 22nd, 2021

DOl: https://doi.org/10.21203/rs.3.rs-151667/v1

License: (9) This work is licensed under a Creative Commons Attribution 4.0 International License. Read Full License 


\section{Abstract}

\section{Background}

Mesenchymal stem cells including adipose stem cells (ASCs) have a huge potential in the field of translational medicine. Unfortunately, multiple factors including older age, co-existing diabetes and obesity may impair cellular function, which hinders the overall effectiveness of autologous stem cell therapy. Noncoding RNAs including microRNAs (miRNA), long ncRNAs (IncRNAs), and circular RNAs (circRNAs) have been demonstrated to play an important role in stem cell biology. However, the whole expression pattern and interaction of these RNAs in ASCs related to diabetes and aging remain unknown.

Method

EdU, transwell and $\beta$-galactosidase staining assays were performed to assess the proliferation, migration and senescence of ASCs isolated from diabetic (D-ASCs), old (O-ASCs), and young (Y-ASCs) donators. The abilities of these ASCs modulating endothelial cells and fibroblasts functions were evaluated by tube formation and wound scratch assays. We conducted high-throughput RNA sequencing (RNA-seq) in these ASCs to uncover the differentially expressed (DE) RNAs. Gene ontology (GO), Kyoto Encyclopedia of Genes and Genomes (KEGG) pathway, and protein-protein interactions (PPI) analyses were performed to interpret the mRNAs with significant differences. The IncRNAs or circRNAs associated competing endogenous RNA (ceRNA) networks were constructed based on the bioinformatic analyses and real-time polymerase chain reaction (RT-PCR) results. The miR-145-5p mimics were transfected into old ASCs and verified by PCR.

Results

ASCs from diabetic and old donators showed inferior migration ability and increased cellular senescence. Besides, old ASCs have decreased capacities for promoting endothelial cells angiogenesis and fibroblasts migration comparing to young ASCs. The DE miRNAs, mRNAs, IncRNAs and circRNAs were successfully identified by RNA-seq in O-ASCs vs Y-ASCs and D-ASCs vs O-ASCs. GO and KEGG analyses demonstrated DE mRNAs were significantly enriched in aging and cell senescence terms separately. PPI networks performed critical DE mRNAs in above groups. MiRNAs with high fold change and low $p$ value were validated by PCR. The four ceRNA networks were conducted based on the bioinformatic analyses and validated miRNAs. The selected mRNAs, IncRNAs and circRNAs in PPI and ceRNA networks were further evaluated by PCR. Then, the ceRNA subnetworks were constructed based on above validated RNAs. In addition, the IncRNA RAET1E-AS1 - miR-145-5p - WNT11/BMPER axis was selected and validated by the PCR and correlation analyses. Finally, overexpression of miR-145-5p could rejuvenate old ASCs phenotype and augment their abilities for modulating endothelial cells and fibroblasts functions.

Conclusion

Taken together, our research may provide new clues to unveil the underlying mechanisms of ASCs dysfunction, and disclose novel targets for restoring their therapeutic properties. 


\section{Background}

Stem cell-based therapies provide another alternative option for tissue regeneration and have been widely studied for the treatment of various human diseases in this century. The latest success of clinical application of limbal stem cells for corneal restoration and transgenic stem cells for epidermis regeneration has given us lots of confidence [1,2], which undoubtedly reflects the huge potential and great prospective in this field. However, majorities of these researches are still under the stage of preclinical or clinical trials. Even though huge investments have been taken, the clinical transformation is still limited owing to lack of efficacy and safety [3]. Actually, there are certain obstacles we must concern when applying stem cells to treat diseases: 1 ) the risk of allosensitization when transplanting allogenic stem cells [4]; 2) intravascular stem cell therapy may trigger instant blood-mediated inflammatory reaction (IBMIR), even thrombosis and embolization [5]; 3) the unfriendly microenvironment of hosts hinders stem cells' vitalities and functionalities [6]; 4) the therapeutic effect of stem cells can be easily impaired by the physical conditions of donators such with high age, diabetes, obesity, chronic kidney diseases, etc [7-9]. Given the high morbidity of diabetes and global aging population, the last obstacle merits more attention.

The dysfunction of stem cells not only causes the failure of tissue maintenance and regeneration where they reside, but also limits their overall effectiveness for repairing other tissues and organs [8, 9]. Adipose stem cells (ASCs) have been recognized as a promising tool for the treatment of many disorders such as nerve injury, skin wound healing, cardiac diseases, autoimmune disorders and so on [10-12]. The function of ASCs primarily relied on their paracrine factors such as growth factors, cytokines, and extracellular vesicles $[13,14]$. However, previous studies demonstrated that diabetic or aged ASCs were compromised in their capacity to promote neovascularization and skin wound healing $[15,16]$. Other researches also showed that diabetes and aging upregulated inflammatory markers and altered the immunomodulatory properties of ASCs $[17,18]$. Besides, the beneficial effects of ASCs on the treatment of critical limb ischemia, diabetes and its' complications have been evidentially impaired by diabetes [19, 20]. These cells showed inferior proliferative and proangiogenic capacities, increased intracellular reactive oxygen species (ROS) accumulation, and exhibited a pro-inflammatory secretome and senescence-associated phenotype compared to those from normal donators [21-23]. Thus, it is quite necessary for us to consider the negative influence of diabetes and aging on ASCs before implantation. Until now, the precise molecular mechanisms of these alterations and impairments in the niche remain largely unknown. Figuring out the underlying mechanisms may give us opportunities to reverse the malfunctions of ASCs for the sake of better stem cell-based therapies.

Regulatory non-coding RNAs have been demonstrated to play an essential role in majorities of cellular processes and associate with various human diseases, which include microRNAs (miRNAs), long noncoding RNAs (IncRNAs), circular RNAs (circRNAs) and so on [24]. Recently studies showed that these RNAs widely anticipate in the proliferation, migration, differentiation, quiescence, and senescence of stem cells $[25,26]$. MiRNAs are small non-coding RNAs (22-26 nucleotides) that can repress gene expression post-transcriptionally [27]. Emerging evidences elucidated that diabetes and aging manipulated the 
expression level of some miRNAs in stem cells, which might weaken their functionalities [22, 28]. Otherwise, modifying the levels of miR-122 [29], miR-5591-5p [30], miR-34a-5p [31] could enhance the therapeutic efficacy of ASCs against liver fibrosis, diabetic wound healing, and ischemic myocardial infarction separately. LncRNAs are a cluster of transcripts with lengths greater than 200 nucleotides, which can function as signals, decoys, guides, and scaffolds [32]. Li et al. and colleagues [33] found that overexpression of IncRNA Bmncr could reverse the damage of aging on bone marrow MSCs (BMMSCs) and promote their ability for bone formation. CircRNAs are covalently closed, endogenous transcripts that exert functions by regulating transcription and protein production, by interfering with splicing or by translating themselves [34]. Lately researches illuminated the important role of circRNAs on diseases such as aging, cancers and diabetes. Moreover, circRNAs and IncRNAs can function as competing endogenous RNAs (ceRNAs), which compete with mRNAs for miRNAs binding, thus promote translation of these mRNAs [35]. The ceRNAs network has been implicated in the functions of stem cells [36]. Thus, what and how non-coding RNAs affect the therapeutic power of ASCs related to diabetes and aging worth to be comprehensively investigated.

In this study, we conducted high-throughput RNA sequencing (RNA-seq) in ASCs isolated from type 2 diabetic, old and young patients to systematically explore dysregulated miRNAs, mRNAs, IncRNAs and circRNAs. As to our knowledge, this is the first time to establish the IncRNAs and circRNAs-associated ceRNAs networks for simultaneously interpreting the poor function of ASCs correlative to diabetes and aging. With the underlying mechanisms unveiled, we think this study will provide theoretical basis and novel methods to restore the function of ASCs, thus lead to better ASCs based therapies in the future.

\section{Methods And Materials}

Cell culture

ASCs were isolated and incubated using our previously described protocols [11]. The patients under skin flap operations were selected from hand surgery department of Wuhan union hospital at the Tongji Medical College of Huazhong University of Science and Technology. These donators were consented at first, and then assigned into three groups: young group (Y-ASCs), old group (O-ASCs), diabetic group (DASCs). The detailed information of these groups was described in Table S1. Human umbilical vein endothelial cells (HUVECs) and human fibroblasts were cultured following our previous protocols [11].

ASCs proliferation and migration

ASCs at passage 4 from above groups were grown in 96-well plates at the same appropriate density. After $48 \mathrm{~h}$ incubation, the proliferative rates of ASCs from each group were evaluated with Cell-Light EdU Apollo in vitro Kit (Ribobio, Guangzhou, China).

The 24-well Transwell Chamber ( $8.0 \mu \mathrm{m}$ pore size, Corning, USA) was used for assessing the migration ability of ASCs from above groups. Briefly, ASCs suspension (20000 cells/well) was added to the upper 
compartment, and incubated in complete culture medium for $24 \mathrm{~h}$. Then, ASCs migrated to the bottom surface were stained with crystal violet staining (Solarbio, Beijing, China) and counted under microscopy.

Senescence-associated $\beta$-galactosidase staining

ASCs (10000 cells/well) at passage 4 from above groups were seeded in 48-well plates. After incubation for 3 days, the activity of senescence-associated $\beta$-Galactosidase was detected using Senescence $\beta$-gal Staining kit (\#9860, CST) according to the manufacturer's instruction.

Wound scratch and tube formation

ASCs from each group were grown in 6-well plates at the same appropriate density for two days. Then the complete culture medium was replaced by the medium without fetal bovine serum. After another day incubation, the medium was collected and considered as the condition medium.

HFs were seeded in 12-well plates and cultured until 100\% confluence. Then the monolayer was scored with a 200 ul sterile pipette tip. After that, the culture medium was replaced with condition media from above groups and maintained for $24 \mathrm{~h}$. The cells were then stained with crystal violet and counted.

HUVECs $(2 \times 104$ cells per well) suspended in condition media were seeded in 96-well plates previously coated with $50 \mu \mathrm{l}$ Matrigel Basement Membrane Matrix (BD Biosciences, CA, USA). After $4 \mathrm{~h}$ incubation, tube formation was detected with the microscopy.

RNA-seq.

ASCs at passage 3 were used for RNA extraction and sequencing. Standard CDNA libraries were conducted and sequenced using the BGISEQ-500 platform and Illumina HiSeq 4000 platform (BGIShenzhen, China). The sequence data were filtered to get clean data, and then mapped to the reference genome with Bowtie2 [37] (version 2.2.5). Further, differential expression analysis was performed using the DEGseq [38]. The expected fragments per kilobase of transcript per million fragments sequenced (FPKM) [39] was used to determine the expression level of mRNAs, IncRNAs and circRNAs. The expression level of miRNAs was determined by the transcript per million (TPM) [39]. The significantly dysregulated RNAs must meet the following criteria: 1) fold changes $\geq 2$ or $\leq-2$; 2) $p$ and q value $<0.001$;

3) TPM value $>=10$ for miRNA or FPKM value $>=0.1$ for other RNAs.

\section{RT-PCR}

Total RNA was isolated with miRNeasy Mini Kit (Qiagen, Germany) according to the manufacturer's instructions. MiRNAs and other RNAs were reversely transcribed into cDNA using the Mir- $\mathrm{X}^{\mathrm{Tm}}$ miRNA FirstStrand Synthesis kit (\#RR638315, TaKaRa) and Prime-Script® RT reagent Kit (\#RR047A, TaKaRa) separately. Real-time PCR was performed on the StepOnePlus ${ }^{\text {TM }}$ platform (Applied Biosystems, USA) using TB Green ${ }^{\circledR}$ Premix Ex Taq ${ }^{\text {TM }}$ II kit (\#RR820A, TaKaRa). The primer sequences were presented in Table S2- 
5. The relative expression levels of targeted genes were calculated using the $2^{-\Delta \Delta C t}$ method and normalized to $\beta$-actin,gapdh or u6.

Western blot

Total proteins were extracted by ripa lysis buffer with proteinase inhibitor (Roche, Switzerland), separated in $10 \%$ SDS-PAGE gel, and then transferred onto PVDF membrane (Millipore, USA). After $1.5 \mathrm{~h}$ blocking with $5 \% \mathrm{w} / \mathrm{v}$ nonfat dry milk buffer, the membrane was incubated overnight with primary antibodies for fibronectin (\#A12932, ABclonal, China), cyclin D1 (\#A11022, ABclonal, China), cyclin A1 (\#ab206746, abcam), nanog (\#14295-1-AP, Proteintech, China), oct4 (\#11263-1-AP, Proteintech, China), p21 (\#10355-1AP, Proteintech, China), gapdh (\#10494-1-AP, Proteintech, China). Then, the membrane was incubated with secondary antibodies (Aspen, China) and exposed to X-ray film (UVP, CA, USA).

CeRNA and PPI network analysis

The significant differentially expressed mRNAs and ncRNAs between each group were used for ceRNA network constructions. The RNAs which could be predicted by at least two of these databases (RNAhybrid [https://bibiserv.cebitec.uni-bielefeld.de/rnahybrid], miRanda [http://www.microrna.org/microrna/home.do] and TargetScan [http://www.targetscan.org]) were considered as the miRNA targets. The sequences of mRNAs, IncRNAs and circRNAs were screened to get the potential MREs. Protein-protein interaction (PPI) analysis of differentially expressed mRNAs was based on the STRING database (https://string-db.org). These networks were illustrated using Cytoscape 3.7.1. The degree centrality of involved genes was calculated by Cytoscan.

GO and KEGG enrichment analyses

The differentially expressed mRNAs were analyzed by Gene Ontology (G0) database and Kyoto Encyclopedia of Genes and Genomes (KEGG) pathway database. The GO categories

(http://geneontology.org) was used for defining the molecular function and biological process involved of candidate genes. The biological function of these genes was further annotated by KEGG database (http://www.genome.jp/kegg). We used the hypergeometric distribution test to find out significantly enriched gene sets. A p value $<0.05$ was considered statistically significant.

Cell transfection

The mimic and inhibitor of hsa-miR-145-5p were designed and synthesized by Ribobio (Guangzhou, China). ASCs were transfected using riboFECT ${ }^{\mathrm{TM}} \mathrm{CP}$ Reagent according to the manufacturer's instruction. The sequences of these oligonucleotides are described below: hsa-miR-145-5p-mimic: 5'GUCCAGUUUUCCCAGGAAUCCCU-3'; mimic-NC: 5'-UUUGUAC UACACAAAAGUACUG-3'; hsa-miR-145-5pinhibitor: 5'-GAGCUACAGUGCUUCAUCUCA-3'; inhibitor-NC: 5'-CAGUACUUUUGUGUAGUACAAA-3'.

Statistics 
All statistical analysis was conducted using Graph Pad prism v 7.0 software. All data were expressed as the mean \pm SEM. The un-paired Student's $t$ test was used for evaluating the statistical discrepancy between two groups. For group $>=3$, one-way ANOVA with Bonferroni post hoc test was employed. The Pearson Correlation Coefficient was used to calculate the expressional correlation of two RNAs. Statistical significance was set at $\mathrm{p}$ or $\mathrm{q}<0.05$.

\section{Results}

ASCs from diabetes or old donators showed inferior proliferation and migration ability, exhibited a senescent phenotype

To figure out whether diabetes and aging affected the phenotype of ASCs, a series of fundamental experiments were conducted. At first, EdU assay showed that the proliferation rate of ASCs isolated from old donators was 1.6-fold lower than that from young donators (Fig. 1A, 1B). Meanwhile, diabetic conditions were not proved to reduce the proliferative ability of ASCs (Fig. 1A, 1B). Then, transwell assays revealed that the migration abilities of ASCs were the best in young group, followed by old group, and diabetic group turns to be the worst (Fig. 1C, 1D). Lastly, $\beta$-galactosidase staining showed the rate of senescent stem cells in diabetic group were significantly higher than that in old group, while ASCs in young group exhibited the minimal senescent cells (Fig. 1E, 1F). In conclusion, diabetes and aging both have somewhat detrimental effects on the phenotype of ASCs.

Diabetic conditions and aging impaired the capacities of ASCs for modulating fibroblasts and HUVECs function

Given above results, we desperately want to know whether diabetic conditions and aging impaired the therapeutic abilities of ASCs. Here we evaluated the differences of ASCs from these three groups in optimizing HUVECs and fibroblasts function. In vitro tube formation assay showed that HUVECs treated with condition media isolated from Y-ASCs exhibited 2 -fold increases of closed tubular structures than that from 0-ASCs (Fig. 1G, 1H). Meanwhile, the number of tubular structures in 0-ASCs group was 2 -fold more than D-ASCs group, but not statistically significant (Fig. 1G, 1H). Besides, wound scratch assay revealed that the migration ability of fibroblasts treated with condition media isolated from Y-ASCs was significantly promoted compared with that from 0-ASCs (Fig. 1I, 1J). In addition, the number of migrative fibroblasts in D-ASCs group was slightly less than 0-ASCs group (Fig. 1I, 1J). Therefore, high age of donators has remarkably deleterious effects on ASCs capacities for modulating cellular functions, while diabetic conditions of donators may have slightly but not significantly harmful effect on ASCs function.

Differential expression analyses: Y-ASCs vs O-ASCs and D-ASCs vs O-ASCs

Having known the unwell impact of diabetes and aging on ASCs phenotype and function, next we performed the RNA-seq analysis on ASCs from these three groups in order to uncover the underlying mechanisms. The differentially expressed miRNAs, mRNAs, IncRNAs and circRNAs from three groups ( $\mathrm{n}$ $=3$ ) were illustrated by the hierarchical clustering heat maps (Fig. 2). A total of 34 (27 up and 7 down) 
differentially expressed miRNAs, 553 (291 up and 262 down) differentially expressed mRNAs, 132 (39 up and 92 down) differentially expressed IncRNAs and 196 (123 up and 73 down) differentially expressed circRNAs were detected between Y-ASCs and O-ASCs (Fig. 2A-D, Data S1-4). Up means RNAs up-regulated in Y-ASCs compared to O-ASCs. Comparing D-ASCs with O-ASCs, we found a total of 43 (24 up and 19 down) differentially expressed miRNAs, 926 (483 up and 443 down) differentially expressed mRNAs, 535 (496 up and 39 down) differentially expressed IncRNAs and 1966 (1806 up and 160 down) differentially expressed circRNAs (Fig. 2E-H, Data S5-8). Up means RNAs up-regulated in D-ASCs compared to O-ASCs. Combining above results, we found 4 miRNAs, 22 mRNAs, 8 circRNAs were up regulated in 0-ASCs compared to D-ASCs, and further up regulated in Y-ASCs compared to O-ASCs (Data S9-11). Moreover, 15 mRNAs, 14 IncRNAs and 17 circRNAs were down regulated in O-ASCs compared to D-ASCs, and further down regulated in Y-ASCs compared to 0-ASCs (Data S10-12).

Functional enrichment analysis of differentially expressed mRNAs

GO and KEGG analyses were conducted to investigate the biological function of dysregulated mRNAs. Comparing Y-ASCs with O-ASCs, GO biological process analysis showed that $750 \mathrm{GO}$ terms were significantly enriched (Data S13). Some terms like aging, positive regulation of cell proliferation, positive regulation of cell migration and angiogenesis may be associated with the dysfunction of ASCs related to donator's age (Fig. 3A). KEGG pathway analysis showed that 47 pathways were statistically enriched (Data S14). Among them, cellular senescence, TGF-beta signaling pathway, p53-signaling pathway and PPAR signaling pathway may be involved in the impaired function of ASCs (Fig. 3B). Comparing 0-ASCs with D-ASCs, 553 GO terms were significantly enriched in GO biological process analysis (Data S15). Some terms such as oxidation-reduction process, aging, cell migration, wound healing and angiogenesis may be associated with the undesirable therapeutic capacity of ASCs exposing to diabetes (Fig. $3 \mathrm{C}$ ). KEGG pathway analysis revealed that 37 pathways were statistically enriched (Data S16). Of them, pathways like AGE-RAGE signaling pathway, TGF-beta signaling pathway, p53-signaling pathway, mTOR signaling pathway, cellular senescence may be participated in the unsatisfactory function of ASCs isolated from diabetic donators (Fig. 3D).

PPI networks construction

PPI networks were constructed in order to identify the critical genes from those differentially expressed mRNAs. Comparing Y-ASCs with O-ASCs, the network was established and compromised of 107 nodes and 373 edges (Fig. 3E). Of them, the top 15 genes with high degree were APOE, BMP4, EGR1, GPC3, CXCL8, SPP1, FOS, HGF, TNC, IGFBP7, JUN, APOL1, GBP2, HLA-F, OAS1 (Fig. 3E). Comparing 0-ASCs with D-ASCs, 131 nodes and 783 edges were included in the network (Fig. 3F). Among them, the top 15 genes with high degree were C3, CXCL8, IL6, CXCL1, CCNB1, BUB1, CXCL12, MCHR2, CXCL2, CXCL6, CXCR4, CXCL5, QSOX1, S1PR1, TAS2R31 (Fig. 3F).

RT-PCR confirmation of differentially expressed miRNAs 
We randomly selected 14 miRNAs to validate the results of RNA-seq data separately. Comparing Y-ASCs with 0-ASCs, miR-145-3P, miR-145-5p, miR-126-3p, miR-126-5p, miR-214-3p, miR-181a-3p, miR-210-3p were identified to be up regulated in Y-ASCs by PCR analysis, which was consistent with the RNA-seq results (Fig. 4A, 4B). Besides, PCR results showed miR-766-3p was down regulated in Y-ASCs, which was opposite with that in the RNA-seq data (Fig. 4A, 4B). Comparing D-ASCs with 0-ASCs, the PCR results of miR-214-3p, miR-193a-3p, miR-3529-3p, miR-145-3p, miR-302a-3p, miR-302b-3p were verified to be statistically significant and consistent with the RNA-seq data (Fig. 4C, 4D). However, the PCR results of two miRNAs (miR-615-3p, miR-12136) were opposite with the RNA-seq data (Fig. 4C, 4D). Other 6 miRNAs which showed no significant difference between these groups by PCR analysis were illustrated in Fig. 4E$\mathrm{F}$.

Construction of circRNA or IncRNA-miRNA-mRNA network

CeRNA networks were constructed based on the differentially expressed RNAs. MiRNAs verified by PCR analysis and consistent with RNA-seq data were selected to be the core of ceRNA networks. Comparing YASCs with O-ASCs, the IncRNA associated ceRNA network was compromised of 4 miRNAs, 171 mRNAs and 46 IncRNAs (Fig. 5A), and the circRNA associated ceRNA network contained 3 miRNAs, 160 mRNAs and 36 circRNAs (Fig. 5B). Comparing D-ASCs with O-ASCs, 4 miRNAs, 118 mRNAs and 97 IncRNAs were included in the IncRNA associated ceRNA network (Fig. 6A), 4 miRNAs, 119 mRNAs and 103 circRNAs were involved in the circRNA associated ceRNA network (Fig. 6B).

RT-PCR confirmation of differentially expressed mRNAs, IncRNAs and circRNAs

MRNAs, IncRNAs and circRNAs contained in above PPI and ceRNA networks were selected for further RTPCR analyses. Comparing Y-ASCs with O-ASCs, RT-PCR results showed 14 mRNAs were significantly dysregulated (Fig. 7A). Among them, MMP3, EGR1, JUNB, BMP4 were involved in the PPI network (Fig. 3E, 7A), ANK2, PODN, NOVA1, CLEC3B, RARRES3, IGFBP6, MFAP5 were involved in the ceRNA network (Fig. 5A-B, 7A), and ITGB8, WNT11, BMPER were involved in both PPI and ceRNA networks (Fig. 3E, 5A-B, 7A). Comparing D-ASCs with O-ASCs, 11 mRNAs were verified to be differentially expressed by PCR analyses (Fig. 7B), of which CXCL8, CXCL1, CXCL6, CXCL5 were all up-regulated in 0-ASCs and involved in the PPI network (Fig. 3F, 7B). Besides, SOX4, ANGPT1, SLC5A3, NDUFV3, LUC7L3 were involved in the ceRNA network (Fig. 6A-B, 7B), and COL18A1, FMOD were involved in both PPI and ceRNA networks (Fig. 3F, 6A-B, 7B). Moreover, we found 5 IncRNAs (RAET1E-AS1, LOC102723591, LOC105373230, LOC112267883, LINC02595) and 5 circRNAs (hsa_circ_0017534, hsa_circ_0092630, hsa_circ_0080906, hsa_circ_0075045, hsa_circ_0080909) were significantly up-regulated in O-ASCs comparing to Y-ASCs (Fig. 7C). Also, the PCR results revealed that 2 IncRNAs (LOC102724087, LOC101928000) and 3 circRNAs (hsa_circ_0088199, hsa_circ_0088195, hsa_circ_0058158) were significantly up-regulated in 0-ASCs comparing to D-ASCs (Fig. 7D). Adversely, 2 IncRNAs (LOC105377989 and NEAT1) were significantly down-regulated in O-ASCs comparing to D-ASCs (Fig. 7D). Other RNAs without statistically significant difference between these groups were displayed in supplementary Figure S1A-B.

Establishment of ceRNAs sub-networks based on the RNAs verified by PCR analyses 
Based on above PCR results, we drew the circRNAs and IncRNAs-miRNAs-mRNAs sub-networks for further investigation. Comparing Y-ASCs with O-ASCs, the network included 5 miRNAs, 10 mRNAs, 5 IncRNAs and 5 circRNAs (Fig. 7E). Comparing D-ASCs with 0-ASCs, 6 miRNAs, 7 mRNAs, 4 IncRNAs and 3 circRNAs were compromised in the network (Fig. 7F). Then, we randomly selected hsa-miR-145-5p for our further study, which had ceRNA relationships with four mRNAs (ITGB8, WNT11, BMPER, RARRES3), two IncRNAs (RAET1E-AS1, LOC102723591) and one circRNA named hsa_circ_0092630 (Fig. 7E). The correlation analyses revealed that the expression levels of RAET1E-AS1, WNT11 and BMPER had significantly negative correlations with that of hsa-miR-145-5p (Fig. 7G-I), while others not (supplementary Figure S1C-D). Meanwhile, the expression level of RAET1E-AS1 had a significantly positive correlation with that of WNT11 and BMPER (Fig. 7J-K). Lastly, we found overexpression of hasmiR-145-5p in ASCs could significantly down-regulated the expression levels of WNT11, BMPER, RAET1EAS1, LOC102723591, hsa_circ_0092630 (Fig. 7L). Therefore, we speculated that the RAET1E-AS1- hsamiR-145-5p-WNT11/BMPER network might play a vital role in the phenotype and function of ASCs.

Overexpression of hsa-miR-145-5p improved the phenotype and function of ASCs, while inhibition of it adversely.

To evaluate the character of the RAET1E-AS1- hsa-miR-145-5p- WNT11/BMPER axis, here we modulated the expression level of hsa-miR-145-5p in ASCs to investigate the changes of their phenotypes and functions. The EdU and transwell assays showed that the proliferative and migrative abilities of ASCs have been promoted more than two-fold after hsa-miR-145-5p overexpression, while inhibition of it significantly reduced these abilities (Fig. 8A-D). Also, the number of $\beta$-galactosidase stained cells in the miR-145-5p group was two-fold less than that of the mimic-NC group, while inhibition of miR-145-5p significantly increased the stained cells (Fig. 8E-F). Next, the in vitro tube formation and wound scratch assays revealed that cellular supernatants of cultured ASCs with miR-145-5p overexpression could remarkably enhance the function of HUVECs and fibroblasts as shown by increases of closed tubular structures and migrative cells, while inhibition of miR-145-5p exhibited the opposite results (Fig. 8G-J). In addition, the western blot analysis showed overexpression of miR-145-5p in ASCs could significantly improve the expression of migration associated protein FN1, proliferation associated proteins CCNA1 and CCND1, pluripotent markers NANOG and OCT4, and decrease the expression of senescence associated protein P21 (Fig. 8K). The PCR analysis showed overexpression of miR-145-5p in ASCs could significantly improve the expression of MMP9, NANOG, growth factors PDGFA and FGF, and decrease the expression of IGF1, HIF, CXCL8, and senescent markers TP53 and CDKN2A (Fig. 8L). In conclusion, overexpression of miR-145-5p in ASCs could ameliorate the unsatisfactory phenotype and function of ASCs isolated from old donators.

\section{Discussion}

Stem cells have occupied an important place in the field of translational medicine, basically through the secretion of trophic factors rather than a direct involvement of tissue reconstruction [8]. Clinical trials often use autologous stem cells to treat human diseases in consideration of immune rejections [4]. 
Unfortunately, patients adaptive for stem cells therapies are often accompanied with systemic diseases, which may threat the functionalities of their own stem cells and raise uncertainty of autologous transplantation [7]. Adipose stem cells have been recognized as one of the most promising stem cell sources in recent years owing to a series of specific advantages: convenient acquisition of adipose tissue, lack of ethical concerns, effortless in vitro isolation and expansion [8]. Unavoidably, the unwell physical conditions of donators such with high age and diabetes impair ASCs' overall benefits and limit their application $[22,23]$. In the present study, we hypothesized that diabetic conditions and aging might change the epigenome, transcriptome, proteome and metabolome of ASCs. Here we mainly focused on the transcriptome and conducted RNA-seq to uncover the underlying mechanisms in this aspect.

Firstly, we assessed the functional and phenotypic differences of ASCs isolated from diabetic, old and young donators. The results were much similar with previous studies. Our study found that ASCs isolated from old donators exhibited impaired proliferative and migration abilities, and were easy to be senescent after in vitro expansion, which were consistent with other studies [21]. Previous studies showed that old ASCs had weakened capacities in accelerating skin wound healing compared to young ASCs, which was similar with our findings that old ASCs had inferior abilities in modulating HUVECs and fibroblasts function [16]. Moreover, our study found that diabetic conditions had no influence on the proliferation of ASCs, which was quite different from most previous studies [20,22]. This discrepancy might due to the differences of original sources, expansion methods and so on. But the migrative and senescent phenotypes of diabetic ASCs in our study were consistent with the previous research [22]. It has been well-documented that diabetic conditions impaired the therapeutic effect of ASCs in wound healing and critical limb ischemia $[19,40]$. Our research also revealed that diabetic ASCs had slightly impaired function for promoting angiogenesis of HUVECs and fibroblasts migration, which might explain the results of those researches. Thus, the physical conditions of donators must be taken into consideration before stem cells therapies.

As is known to us, the pathogenesis and progress of majorities diseases such as cancers and metabolic diseases are often accompanied with the transcriptome alteration. Non-coding RNAs, as vital members of transcriptome, have been verified to participate in the physiopathologic mechanisms of those diseases $[24,41]$. Previous studies have shown the different expression levels of miRNAs and IncRNAs by microarrays in BMMSCs isolated from young and old donators $[42,43]$. However, the whole transcriptome alteration of ASCs related to diabetes and aging remains to be investigated. In the present study, we simultaneously explored the miRNAs, mRNAs, IncRNAs and circRNAs expression differences in ASCs isolated from diabetic, old and young donators by RNA-sEq. Our study found 34 miRNAs, 553 mRNAs, 132 IncRNAs, 196 circRNAs were differentially expressed between Y-ASCs and O-ASCs, and 43 miRNAs, 926 mRNAs, 535 IncRNAs, 1966 circRNAs were differentially expressed between D-ASCs and O-ASCs. GO analyses showed biological processes like aging, angiogenesis, cell adhesion, positive regulation of cell proliferation, cell-cell signaling were both enriched in two comparison. KEGG analyses revealed AGERAGE signaling pathway, cellular senescence, p53 signaling pathway were associated with those two influence factors. Also, p53 signaling pathway have been well-documented to involve in the proliferation, senescence, apoptosis and differentiation of stem cells [44].

Page 12/31 
Moreover, we conducted PPI networks in order to further analyze the differentially expressed mRNAs. Our results showed 107 genes were involved in the network comparing Y-ASCs with O-ASCs. Of them, MMP3, EGR1, JUNB, BMP4, ITGB8, WNT11, BMPER were verified by PCR analyses. Recent study revealed that induction of EGR1 could trigger glioblastomas cells dedifferentiation into a stem-like state, expressing pluripotent markers NANOG and OCT4 [45]. JUNB, a member of the AP-1 transcription factor family, has been verified to regulate epidermal stem cells through balancing proliferation and differentiation of progenitors [46]. BMP4, as a member of the BMP-SMAD signaling axis, could suppress $\mathrm{p} 16$ /INK4Amediated cell senescence [47]. EGR1, JUNB and BMP4 were all down-regulated in 0-ASCs comparing with Y-ASCs in our study, which might account for the dysfunction of ASCs in old group. In addition, we also found 131 genes were involved in the PPI network comparing D-ASCs with O-ASCs. Among them, PCR analyses showed CXCL8, CXCL1, CXCL6, CXCL5, COL18A1, FMOD were all down-regulated in D-ASCs comparing with O-ASCs. These results were quite different from previous study showing ASCs from patients with diabetes or atherosclerosis highly expressed pro-inflammatory markers like IL-1区, IL6, IL$8 / C X C L 8[17,18]$. However, recent study also elucidated that overexpression of dual chemokine CXCL6 and SDF-1】 in ASCs could promote their angio-vasculogenic property [48]. Since the multiple functions of these chemokines [49], the specific roles of them in ASCs need to be further evaluated.

CeRNA network have been described as an intricate interplay among diverse RNA species, in which mRNAs, IncRNAs, pseudogenes and circRNAs compete for binding to shared miRNAs [35]. This crosstalk has been clarified to participate in many biological process and human diseases, especially in oncogenesis and progression [50]. Few attentions have been paid in the present field. Previous study showed LINC00707 could promote osteogenesis of human BMMSCs by acting as a ceRNA to upregulate WNT2B via miR-370-3p inhibition [51]. CircFOXP1 could sustain mesenchymal stem cell identity and differentiation via competing with WNT5A for miR-17-3p/miR-127-5p binding [36]. Since miRNAs are the core of the ceRNA network, we at first evaluated the differentially expressed miRNAs in RNA-seq data by PCR analyses. Our results revealed eight miRNAs (miR-145-3P, miR-145-5p, miR-126-3p, miR-126-5p, miR-214-3p, miR-181a-3p, miR-210-3p, miR-766-3p) were differentially expressed between Y-ASCs and 0ASCs. Of them, miR-210-3p [52] and miR-126 [53] could inhibit the apoptosis of ASCs and BMMSCs, miR214-3p could anticipate in the therapeutic function of MSCs derived extracellular vesicles for attenuating radiation-induced lung injury [54]. However, all these three miRNAs were down-regulated in 0-ASCs, which might be part of the reasons for their poor function. Otherwise, we also found eight miRNAs (miR-214-3p, miR-193a-3p, miR-3529-3p, miR-145-3p, miR-302a-3p, miR-302b-3p, miR-615-3p, miR-12136) were differentially expressed between D-ASCs and 0-ASCs. Among them, miR-302 clusters were highly expressed in human embryonic stem cells and associated with stem cell pluripotency [55]. Surprisedly, most of above miRNAs have not been fully reported in the field of mesenchymal stem cells' phenotypes and functions. Thus, further study should be done to investigate the characters of them in this field.

Next, we constructed ceRNA networks using bioinformatics methods based on the differentially expressed mRNAs, IncRNAs, circRNAs and verified miRNAs. The four network maps illustrated lots of possible ceRNA relationships which might play vital roles in ASCs biology. Part of RNAs in the networks were then selected and analyzed by PCR in order to establish the sub-networks. The two sub-networks 
included 9 miRNAs, 17 mRNAs, 9 IncRNAs and 8 circRNAs. Most of involved protein genes locate in extracellular region, and some of them like COL18A1, MFAP5, ITGB8, FMOD and PODN are extracellular matrix related proteins. SOX4 and ANGPT1, well-known genes for stemness and angiogenesis, were down regulated in D-ASCs in our study. Liu et al. and colleagues [56] also illuminated that BMMSCs transfected with ANGPT1 had therapeutic effects on hyperoxia induced optic nerve injury. As for non-coding RNAs in the networks, NEAT1 have been proven to act as an oncogene in multiple cancers via binding various miRNAs $[57,58]$. LINC02595 could promote colorectal cancer progression by inhibiting miR-203b-3p activity [59]. Unexpectedly, the biological function of other IncRNAs and all above circRNAs has not been reported until now. The RAET1E-AS1 - miR-145-5p - WNT11/BMPER axis was selected and conformed based on the ceRNA relationship and expressional correlation analyses. MiR-145-5p have been recognized as a tumor repressor in many kinds of cancers [60,61], a suppressor in osteogenic differentiation of ASCs [62], and a biomarker for the diagnosis of type two diabetes because of the low level in diabetic plasma [63]. Our study found overexpression of miR-145-5p in 0-ASCs could promote their proliferation and migration and rejuvenate their senescent phenotype, which was contrary to their behavior in cancer cells. These results suggest that the tissue specificity of miRNAs should be considered firstly before defining their biological function. In addition, we also revealed overexpression of miR-145-5p could enhance O-ASCs capacities for optimizing HUVECs and fibroblasts function. Thus, modulation of miR-145-5p in ASCs might be a promising method for promoting stem cell function and further investigation should be done to explore the therapeutic effect of modified ASCs in animal model. Chen et al. and colleagues [64] found WNT11 overexpression could inhibit ASCs proliferation and induced them differentiation into nucleus pulposus cells and osteoblasts. Pérez et al. and colleagues [65] found BMPER was up-regulated in diet- and obese-derived ASCs and could decrease their migration ability. However, the role of IncRNA RAET1E-AS1 in cell biology has never been explored. Further fundamental study should be done to investigate the specific function of RAET1E-AS1/ WNT11/ BMPER in ASCs and their direct combination with miR-145-5p.

\section{Conclusion}

In the present study, our group simultaneously examined the changing expression of miRNAs, mRNAs, IncRNAs and circRNAs in ASCs associated with diabetes and aging. GO and KEGG pathway analyses were conducted to annotate the possible function of the differentially expressed mRNAs. PPI networks were established in order to find critical protein genes highly involved in our disease models. The ceRNA networks including IncRNA-miRNA-mRNA and cirRNA-miRNA-mRNA interactions were successfully constructed based on the bioinformatic analyses and PCR results. Overexpression of miR-145-5p could rejuvenate old ASCs phenotype and augment their abilities for modulating endothelial cells and fibroblasts functions (Fig. 9). Thus, this study may contribute to our understanding of the underlying mechanisms of ASCs instability and provide novel targets to reverse the dysfunction of ASCs isolated from diabetic and old patients.

\section{Abbreviations}


AGE: advanced glycation end products; ANGPT1: angiopoietin 1; ANK2: ankyrin 2; APOE: apolipoprotein E; APOL1: apolipoprotein L1; ASCs: adipose stem cells; BMMSCs: bone marrow mesenchymal stem cells; BMP4: bone morphogenetic protein 4; BMPER: BMP binding endothelial regulator; C3: complement C3; CCNA1: cyclin A1; CCNB1: cyclin B1; CCND1: cyclin D1; CDKN2A: cyclin dependent kinase inhibitor 2A; ceRNA: competing endogenous RNA; circRNA: circular RNA; CLEC3B: C-type lectin domain family 3 member B; COL18A1: collagen type XVIII alpha 1 chain; CXCL1: C-X-C motif chemokine ligand 1; CXCL12: C-X-C motif ligand 12; CXCL2: C-X-C motif chemokine ligand 2; CXCL5: C-X-C motif chemokine ligand 5; CXCL6: C-X-C motif chemokine ligand 6; CXCL8: C-X-C motif ligand 8; CXCR4: C-X-C motif chemokine receptor 4; EGR1: Early growth response protein 1; FGF: fibroblast growth factor; FMOD: fibromodulin; FPKM: fragments per kilobase of transcript per million fragments sequenced; GBP2: guanylate binding protein 2; GO: Gene ontology; GPC3: Gypican3; HGF: hepatocyte growth factor; HIF: hypoxia inducible factor; HLA-F: major histocompatibility complex, class I, F; HUVECs: Human umbilical vein endothelial cells; IBMIR: instant blood-mediated inflammatory reaction; IGF1: insulin like growth factor 1; IGFBP6: insulin like growth factor binding protein 6; IGFBP7: insulin-like growth factor binding protein 7; IL6: interleukin 6; ITGB8: integrin subunit beta 8; KEGG: Kyoto Encyclopedia of Genes and Genomes; IncRNA: long non-coding RNA; LUC7L3: LUC7 like 3 pre-mRNA splicing factor; MCHR2: melanin concentrating hormone receptor 2; MFAP5: microfibril associated protein 5; miRNA: microRNA; MMP9: matrix metallopeptidase 9; NDUFV3: NADH ubiquinone oxidoreductase subunit V3; NEAT1: nuclear paraspeckle assembly transcript 1; NOVA1: NOVA alternative splicing regulator 1; OAS1: 2'-5'-oligoadenylate synthetase 1; OCT4: organic cation/carnitine transporter4; PDGFA: platelet derived growth factor subunit A; PODN: podocan; PPAR: peroxisome proliferators-activated receptor; PPI: protein-protein interaction; QSOX1: quiescin sulfhydryl oxidase 1; RAET1E-AS1: RAET1E antisense RNA 1; RAGE: receptor for advanced glycation end products; RARRES3: retinoic acid receptor responder 3; ROS: reactive oxygen species; RT-PCR: real-time polymerase chain reaction; S1PR1: sphingosine-1-phosphate receptor 1; SLC5A3: solute carrier family 5 member 3; SOX4: SRY-box transcription factor 4; SPP1: secreted phosphoprotein 1; TAS2R31: taste 2 receptor member 31; TNC: tenascin C.

\section{Declarations}

\section{Acknowledgments}

Not applicable.

\section{Funding}

This work was supported by the National Natural Science Foundation of China (Grant No. 81772094, 81974289) and the Fundamental Research Funds for the Central Universities (HUST. 2019JYCXJJ051).

\section{Availability of data and materials}

Not applicable. 


\section{Authors' contributions}

SR: conception and design, collection and/or assembly of data, data analysis and interpretation, and manuscript writing. HX: collection and/or assembly of data, isolation of adipose stem cells and in vivo experimental tissue harvest. YL, ZX and XY: study materials provision. JC, JG, TJ, MY, YL, NZ and HC: isolation of adipose stem cells and in vivo experimental tissue harvest. HGM and WL: manuscript revision. ZC: conception and design, financial support, and final approval of manuscript. All authors read and approved the final manuscript.

\section{Ethics approval and consent to participate}

The use of human adipose tissue and foreskin was approved by the Ethics Committee of Tongji Medical College, Huazhong University of Science and Technology. All the patients have been informed in advance and consented to participate in this study.

\section{Consent for publication}

Not applicable.

\section{Competing interests}

The authors declare that they have no competing interests.

\section{Author details}

${ }^{1}$ Department of Hand Surgery, Union Hospital, Tongji Medical College, Huazhong University of Science and Technology, Wuhan, China. ${ }^{2}$ Department of Pathology, Union Hospital, Tongji Medical College, Huazhong University of Science and Technology, Wuhan, China. ${ }^{3}$ Department of Hand and Foot Surgery, Huazhong University of Science and Technology Union Shenzhen Hospital, Shenzhen, Guangdong, China. ${ }^{4}$ Department of Plastic and Hand Surgery, Technical University of Munich, Munich, Germany.

\section{References}

1. Blau HM, Daley GQ. Stem Cells in the Treatment of Disease. N Engl J Med. 2019;380:1748-60.

2. Cossu G, Birchall M, Brown T, De Coppi P, Culme-Seymour E, Gibbon S, et al. Lancet Commission: Stem cells and regenerative medicine. The Lancet. 2018;391:883-910.

3. Trounson A, McDonald C. Stem Cell Therapies in Clinical Trials: Progress and Challenges. Cell Stem Cell. 2015;17:11-22.

4. Welsch CA, Rust WL, Csete M. Concise Review: Lessons Learned from Islet Transplant Clinical Trials in Developing Stem Cell Therapies for Type 1 Diabetes. Stem Cells Transl Med. 2019;8:209-14.

5. Moll G, Ankrum JA, Kamhieh-Milz J, Bieback K, Ringdén O, Volk H, et al. Intravascular Mesenchymal Stromal/Stem Cell Therapy Product Diversification: Time for New Clinical Guidelines. Trends Mol 
Med. 2019;25:149-63.

6. Mazini L, Rochette L, Admou B, Amal S, Malka G. Hopes and Limits of Adipose-Derived Stem Cells (ADSCs) and Mesenchymal Stem Cells (MSCs) in Wound Healing. Int J Mol Sci. 2020;21:1306.

7. Hickson LJ, Eirin A, Lerman LO. Challenges and opportunities for stem cell therapy in patients with chronic kidney disease. Kidney Int. 2016;89:767-78.

8. Duscher D, Barrera J, Wong VW, Maan ZN, Whittam AJ, Januszyk M, et al. Stem Cells in Wound Healing: The Future of Regenerative Medicine? A Mini-Review. Gerontology. 2016;62:216-25.

9. Louwen F, Ritter A, Kreis NN, Yuan J. Insight into the development of obesity: functional alterations of adipose-derived mesenchymal stem cells. Obes Rev. 2018;19:888-904.

10. Kang Y, Liu Y, Liu Z, Ren S, Xiong H, Chen J, et al. Differentiated human adipose-derived stromal cells exhibit the phenotypic and functional characteristics of mature Schwann cells through a modified approach. Cytotherapy. 2019;21:987-1003.

11. Ren S, Chen J, Duscher D, Liu Y, Guo G, Kang Y, et al. Microvesicles from human adipose stem cells promote wound healing by optimizing cellular functions via AKT and ERK signaling pathways. Stem Cell Res Ther. 2019;10.

12. Ceccarelli S, Pontecorvi P, Anastasiadou E, Napoli C, Marchese C. Immunomodulatory Effect of Adipose-Derived Stem Cells: The Cutting Edge of Clinical Application. Frontiers in Cell and Developmental Biology. 2020;8.

13. Chen J, Ren S, Duscher D, Kang Y, Liu Y, Wang C, et al. Exosomes from human adipose-derived stem cells promote sciatic nerve regeneration via optimizing Schwann cell function. J Cell Physiol. 2019.

14. Shukla L, Yuan Y, Shayan R, Greening DW, Karnezis T. Fat Therapeutics: The Clinical Capacity of Adipose-Derived Stem Cells and Exosomes for Human Disease and Tissue Regeneration. Front Pharmacol. 2020;11.

15. Rennert RC, Sorkin M, Januszyk M, Duscher D, Kosaraju R, Chung MT, et al. Diabetes impairs the angiogenic potential of adipose-derived stem cells by selectively depleting cellular subpopulations. Stem Cell Res Ther. 2014;5:79.

16. Duscher D, Rennert RC, Januszyk M, Anghel E, Maan ZN, Whittam AJ, et al. Aging disrupts cell subpopulation dynamics and diminishes the function of mesenchymal stem cells. Sci Rep. 2014;4:7144.

17. Serena C, Keiran N, Ceperuelo-Mallafre V, Ejarque M, Fradera R, Roche K, et al. Obesity and Type 2 Diabetes Alters the Immune Properties of Human Adipose Derived Stem Cells. Stem Cells. 2016;34:2559-73.

18. Kizilay MO, Lora M, Shum-Tim D, Nadeau S, Rodier F, Colmegna I. A Proinflammatory Secretome Mediates the Impaired Immunopotency of Human Mesenchymal Stromal Cells in Elderly Patients with Atherosclerosis. Stem Cells Transl Med. 2017;6:1132-40.

19. Inoue O, Usui S, Takashima S, Nomura A, Yamaguchi K, Takeda Y, et al. Diabetes impairs the angiogenic capacity of human adipose-derived stem cells by reducing the CD271+ subpopulation in adipose tissue. Biochem Bioph Res Co. 2019;517:369-75. 
20. Wang M, Song L, Strange C, Dong X, Wang H. Therapeutic Effects of Adipose Stem Cells from Diabetic Mice for the Treatment of Type 2 Diabetes. Mol Ther. 2018;26:1921-30.

21. Marędziak M, Marycz K, Tomaszewski KA, Kornicka K, Henry BM. The Influence of Aging on the Regenerative Potential of Human Adipose Derived Mesenchymal Stem Cells. Stem Cells Int. 2016;2016:1-15.

22. Alicka M, Major P, Wysocki M, Marycz K. Adipose-Derived Mesenchymal Stem Cells Isolated from Patients with Type 2 Diabetes Show Reduced "Stemness" through an Altered Secretome Profile, Impaired Anti-Oxidative Protection, and Mitochondrial Dynamics Deterioration. J Clin Med. 2019;8:765.

23. Alicka M, Kornicka-Garbowska K, Kucharczyk K, Kępska M, Röcken M, Marycz K. Age-dependent impairment of adipose-derived stem cells isolated from horses. Stem Cell Res Ther. 2020;11.

24. Slack FJ, Chinnaiyan AM. The Role of Non-coding RNAs in Oncology. Cell. 2019;179:1033-55.

25. Shenoy A, Blelloch RH. Regulation of microRNA function in somatic stem cell proliferation and differentiation. Nat Rev Mol Cell Bio. 2014;15:565-76.

26. Hu S, Shan G. LncRNAs in Stem Cells. Stem Cells Int. 2016;2016:1-8.

27. Bartel DP. MicroRNAs: Target Recognition and Regulatory Functions. Cell. 2009;136:215-33.

28. Choi SW, Lee JY, Kang K. miRNAs in stem cell aging and age-related disease. Mech Ageing Dev. 2017;168:20-9.

29. Lou G, Yang Y, Liu F, Ye B, Chen Z, Zheng M, et al. MiR-122 modification enhances the therapeutic efficacy of adipose tissue-derived mesenchymal stem cells against liver fibrosis. J Cell Mol Med. 2017;21:2963-73.

30. Li Q, Xia S, Yin Y, Guo Y, Chen F, Jin P. miR-5591-5p regulates the effect of ADSCs in repairing diabetic wound via targeting AGEs/AGER/JNK signaling axis. Cell Death Dis. 2018;9:566.

31. Weng C, Wu C, Kao S, Chen J, Lin H. Down-Regulation of miR-34a-5p Potentiates Protective Effect of Adipose-Derived Mesenchymal Stem Cells Against Ischemic Myocardial Infarction by Stimulating the Expression of C1q/Tumor Necrosis Factor-Related Protein-9. Front Physiol. 2019;10.

32. Ponting CP, Oliver PL, Reik W. Evolution and Functions of Long Noncoding RNAs. Cell. 2009;136:62941.

33. Li C, Xiao Y, Yang M, Su T, Sun X, Guo Q, et al. Long noncoding RNA Bmncr regulates mesenchymal stem cell fate during skeletal aging. The Journal of clinical investigation. 2018.

34. Kristensen LS, Andersen MS, Stagsted LVW, Ebbesen KK, Hansen TB, Kjems J. The biogenesis, biology and characterization of circular RNAs. Nature reviews. Genetics. 2019;20:675-91.

35. Tay Y, Rinn J, Pandolfi PP. The multilayered complexity of ceRNA crosstalk and competition. Nature. 2014;505:344-52.

36. Cherubini A, Barilani M, Rossi RL, Jalal MMK, Rusconi F, Buono G, et al. FOXP1 circular RNA sustains mesenchymal stem cell identity via microRNA inhibition. Nucleic Acids Res. 2019.

37. Langmead B, Salzberg SL. Fast gapped-read alignment with Bowtie 2. Nat Methods. 2012;9:357-9. 
38. Wang L, Feng Z, Wang X, Wang X, Zhang X. DEGseq: an R package for identifying differentially expressed genes from RNA-seq data. Bioinformatics. 2010;26:136-8.

39. Li B, Dewey CN. RSEM: accurate transcript quantification from RNA-Seq data with or without a reference genome. Bmc Bioinformatics. 2011;12:323.

40. Cianfarani F, Toietta G, Di Rocco G, Cesareo E, Zambruno G, Odorisio T. Diabetes impairs adipose tissue-derived stem cell function and efficiency in promoting wound healing. Wound Repair Regen. 2013;21:545-53.

41. Sallam T, Sandhu J, Tontonoz P. Long Noncoding RNA Discovery in Cardiovascular Disease. Circ Res. 2018;122:155-66.

42. Li CJ, Xiao Y, Yang M, Su T, Sun X, Guo Q, et al. Long noncoding RNA Bmncr regulates mesenchymal stem cell fate during skeletal aging. J Clin Invest. 2018;128:5251-66.

43. Li J, Dong J, Zhang Z, Zhang D, You X, Zhong Y, et al. miR-10a restores human mesenchymal stem cell differentiation by repressing KLF4. J Cell Physiol. 2013;228:2324-36.

44. Fu X, Wu S, Li B, Xu Y, Liu J. Functions of p53 in pluripotent stem cells. Protein Cell. 2020;11:71-8.

45. Almairac F, Turchi L, Sakakini N, Debruyne DN, Elkeurti S, Gjernes E, et al. ERK-Mediated Loss of miR199a-3p and Induction of EGR1 Act as a "Toggle Switch" of GBM Cell Dedifferentiation into NANOGand OCT4-Positive Cells. Cancer Res. 2020;80:3236-50.

46. Singh K, Camera E, Krug L, Basu A, Pandey RK, Munir S, et al. JunB defines functional and structural integrity of the epidermo-pilosebaceous unit in the skin. Nat Commun. 2018;9.

47. Hayashi Y, Hsiao EC, Sami S, Lancero M, Schlieve CR, Nguyen T, et al. BMP-SMAD-ID promotes reprogramming to pluripotency by inhibiting p16/INK4A-dependent senescence. Proceedings of the National Academy of Sciences. 2016;113:13057-62.

48. Min Y, Han S, Aae Ryu H, Kim S. Human adipose mesenchymal stem cells overexpressing dual chemotactic gene showed enhanced angiogenic capacity in ischaemic hindlimb model. Cardiovasc Res. 2018;114:1400-9.

49. Morein D, Erlichman N, Ben-Baruch A. Beyond Cell Motility: The Expanding Roles of Chemokines and Their Receptors in Malignancy. Front Immunol. 2020;11.

50. Zhu K, Zhang C, Ma X, Hu J, Cai T, Zhang L. Analyzing the Interactions of mRNAs and ncRNAs to Predict Competing Endogenous RNA Networks in Osteosarcoma Chemo-Resistance. Mol Ther. 2019;27:518-30.

51. Jia B, Wang Z, Sun X, Chen J, Zhao J, Qiu X. Long noncoding RNA LINC00707 sponges miR-370-3p to promote osteogenesis of human bone marrow-derived mesenchymal stem cells through upregulating WNT2B. Stem Cell Res Ther. 2019;10.

52. Lai CH, Barik P, Hsieh DJ, Day CH, Ho TJ, Chen RJ, et al. Inhibition of cell death-inducing p53 target 1 through miR-210-3p overexpression attenuates reactive oxygen species and apoptosis in rat adiposederived stem cells challenged with Angiotensin II. Biochem Biophys Res Commun. 2020. 
53. Kong R, Gao J, Ji L, Zhao D. MicroRNA-126 promotes proliferation, migration, invasion and endothelial differentiation while inhibits apoptosis and osteogenic differentiation of bone marrowderived mesenchymal stem cells. Cell Cycle. 2020;19:2119-38.

54. Lei X, He N, Zhu L, Zhou M, Zhang K, Wang C, et al. Mesenchymal Stem Cell-Derived Extracellular Vesicles Attenuate Radiation-Induced Lung Injury via miRNA-214-3p. Antioxid Redox Signal. 2020.

55. Gu KL, Zhang Q, Yan Y, Li TT, Duan FF, Hao J, et al. Pluripotency-associated miR-290/302 family of microRNAs promote the dismantling of naive pluripotency. Cell Res. 2016;26:350-66.

56. Liu FY, Li GW, Sun CH, Chen S, Cao JF, Ma QQ, et al. Effects of bone marrow mesenchymal stem cells transfected with Ang-1 gene on hyperoxia-induced optic nerve injury in neonatal mice. J Cell Physiol. 2018;233:8567-77.

57. Liu X, Yao W, Xiong H, Li Q, Li Y. LncRNA NEAT1 accelerates breast cancer progression through regulating miR-410-3p/ CCND1 axis. Cancer Biomark. 2020.

58. Bu FT, Wang A, Zhu Y, You HM, Zhang YF, Meng XM, et al. LncRNA NEAT1: Shedding light on mechanisms and opportunities in liver diseases. Liver Int. 2020.

59. Yang Z, An Y, Wang N, Dong X, Kang H. LINC02595 promotes tumor progression in colorectal cancer by inhibiting miR-203b-3p activity and facilitating BCL2L1 expression. J Cell Physiol. 2020;235:744964.

60. Zhang Z, Wang C, Zhang Y, Yu S, Zhao G, Xu J. CircDUSP16 promotes the tumorigenesis and invasion of gastric cancer by sponging miR-145-5p. Gastric Cancer. 2020;23:437-48.

61. Chen J, Chen T, Zhu Y, Li Y, Zhang Y, Wang Y, et al. circPTN sponges miR-145-5p/miR-330-5p to promote proliferation and stemness in glioma. J Exp Clin Canc Res. 2019;38.

62. Yu L, Qu H, Yu Y, Li W, Zhao Y, Qiu G. LncRNA-PCAT1 targeting miR-145-5p promotes TLR4associated osteogenic differentiation of adipose-derived stem cells. J Cell Mol Med. 2018;22:613447.

63. Shahrokhi SZ, Saeidi L, Sadatamini M, Jafarzadeh M, Rahimipour A, Kazerouni F. Can miR-145-5p be used as a marker in diabetic patients? Arch Physiol Biochem. 2020:1-6.

64. Chen $\mathrm{H}$, Huang A, He Y, Bian J, Li H. Wnt11 overexpression promote adipose-derived stem cells differentiating to the nucleus pulposus-like phenotype. Eur Rev Med Pharmaco. 2017;21:1462.

65. Pérez LM, de Lucas B, Gálvez BG. BMPER is upregulated in obesity and seems to have a role in pericardial adipose stem cells. J Cell Physiol. 2020.

\section{Figures}



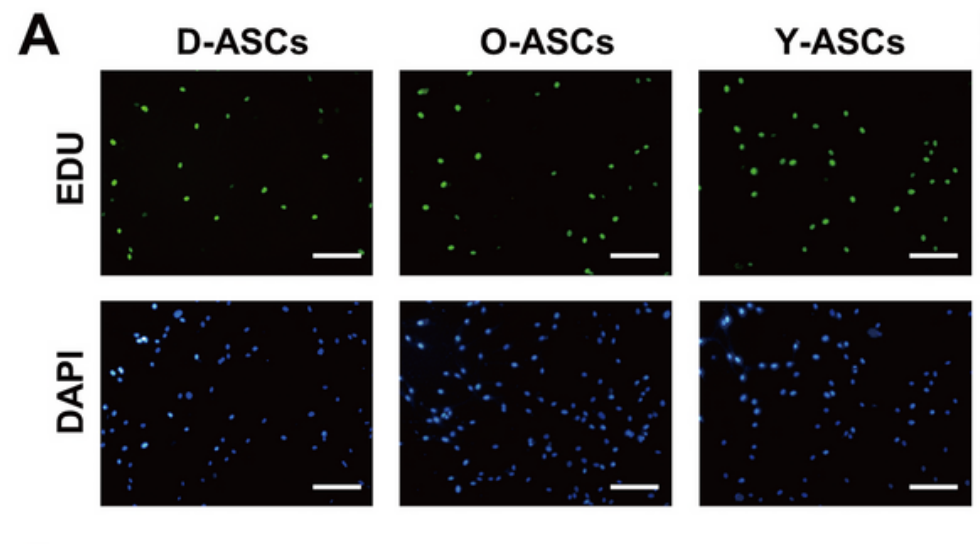

B
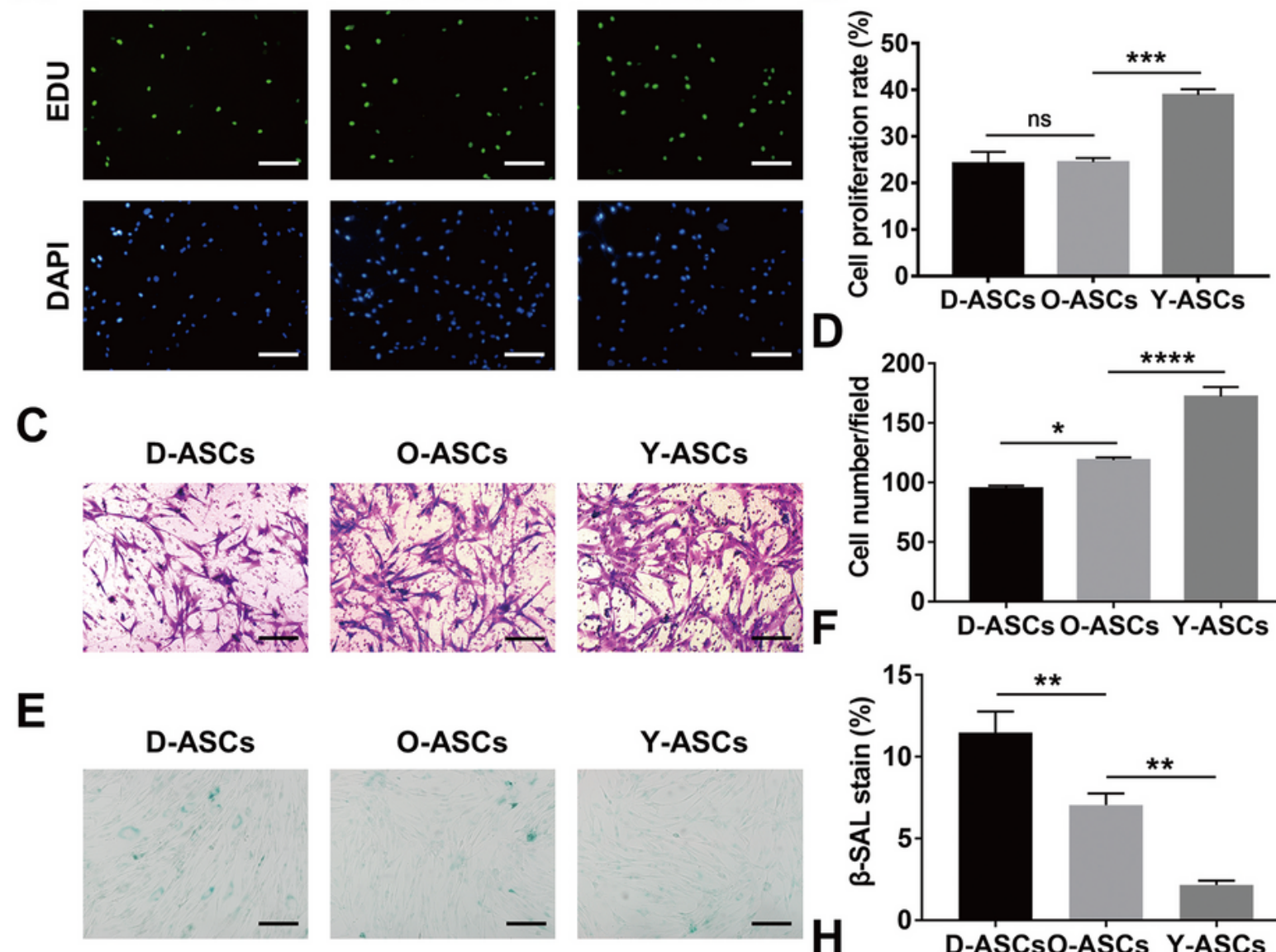

O-ASCs

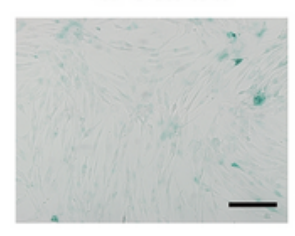

Y-ASCs

G

D-ASCs

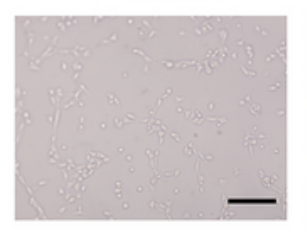

D-ASCs

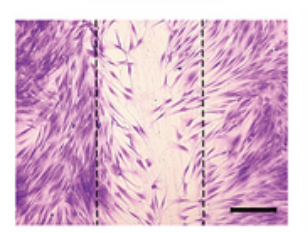

O-ASCs

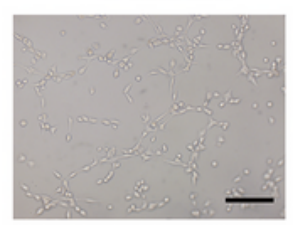

O-ASCs

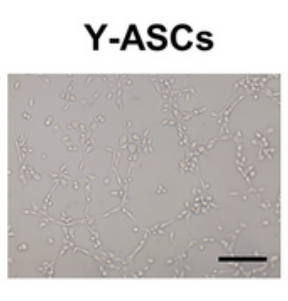

Y-ASCs
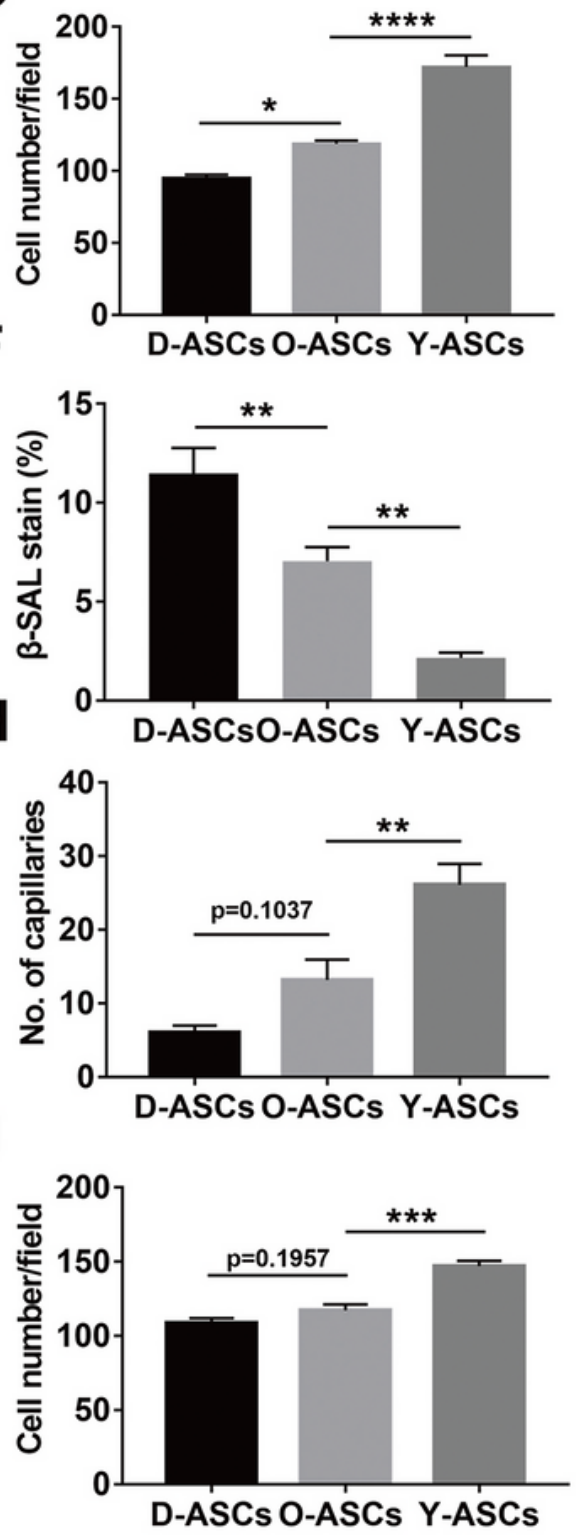

Figure 1

Evaluation of the phenotype and function of ASCs isolated from diabetic, old and young patients. (A) EdU assay analysis of the proliferative rate of ASCs from those three groups. the proliferative cells and cellular nuclei were stained with green and blue colors. Scar bar: $50 \mu \mathrm{m}$. (C) Images of migrated ASCs from those three groups. Scar bar: $50 \mu \mathrm{m}$. (E) $\beta$-gal Staining assay analysis of the senescent rate of ASCs from those three groups. The senescent cells were stained with the green color. Scar bar: $50 \mu \mathrm{m}$. (G) 
Images of tube formations in HUVECs treated with conditional media of ASCs from those three groups. Scar bar: $50 \mu \mathrm{m}$. (I) Images of migrated fibroblasts given above treatments. Scar bar: $100 \mu \mathrm{m}$. (B, D, F, H, J) qualified data shown in (A, C, E, G, I) separately. $N=5 .{ }^{*} p<0.05,{ }^{* *} p<0.01$, ${ }^{* *} p<0.001,{ }^{* * * *} p<0.0001$.

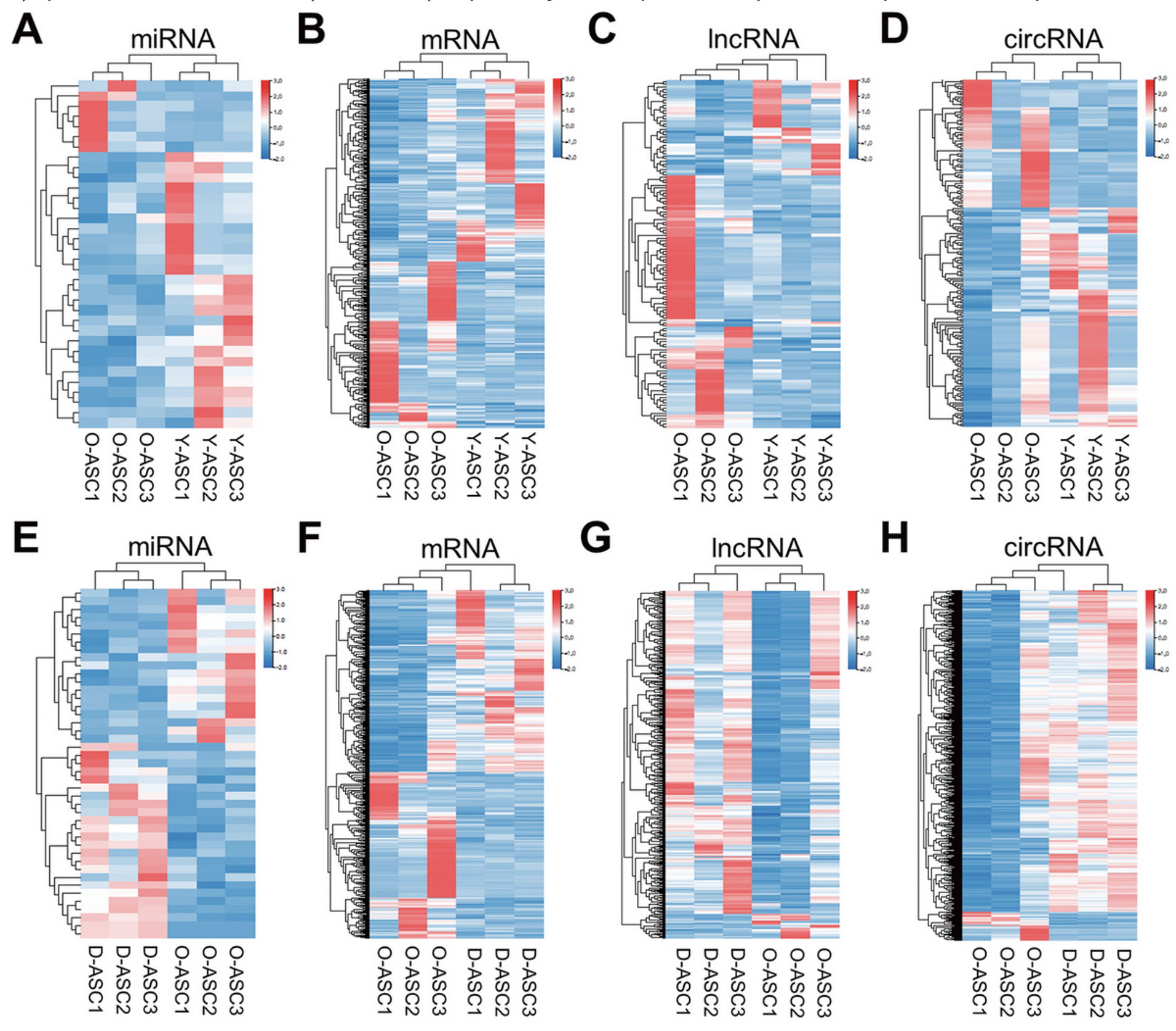

Figure 2

Expression profiles of RNAs comparing O-ASCs with Y-ASCs and D-ASCs with O-ASCs. the miRNAs, mRNAs, IncRNAs and circRNAs profiles, comparing O-ASCs with Y-ASCs were shown in heatmap (A-D), comparing D-ASCs with O-ASCs were shown in heatmap (E-H). N=3. 

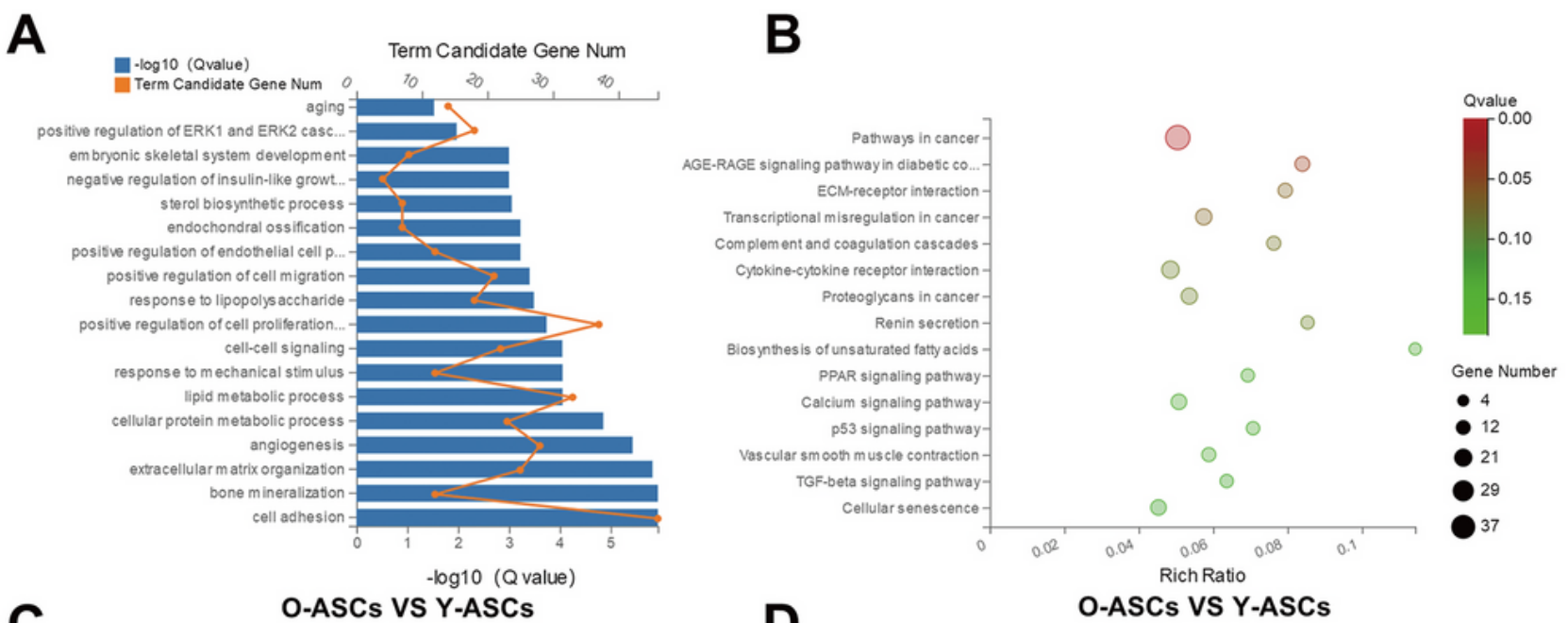

C

O-ASCs VS Y-ASCs

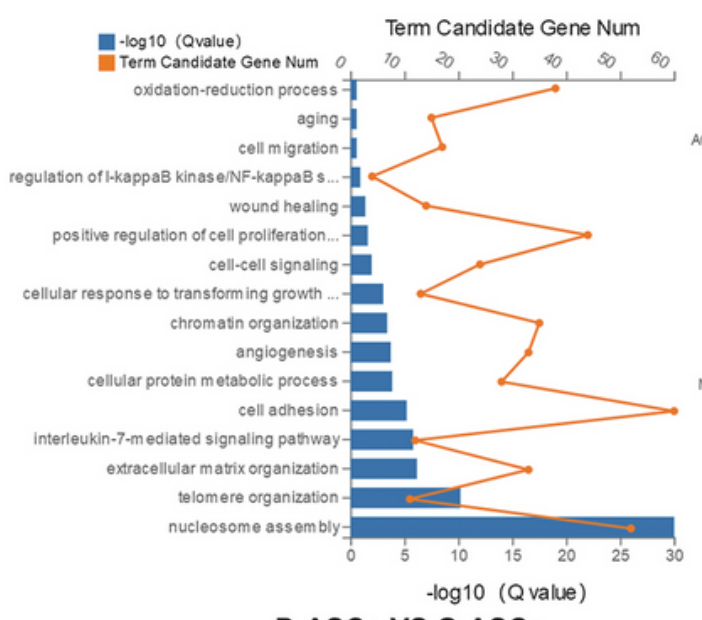

D

O-ASCs VS Y-ASCs

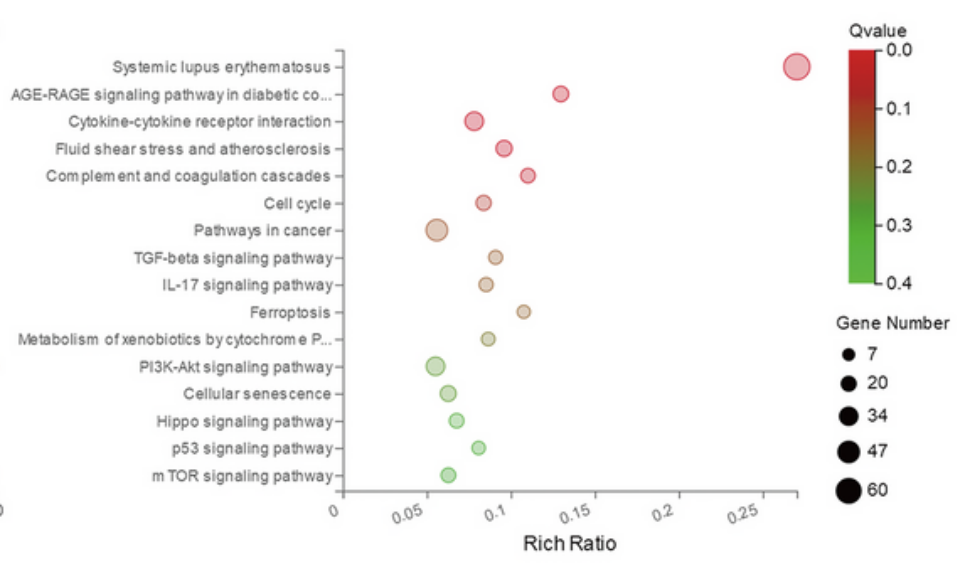

D-ASCs VS O-ASCs

D-ASCs VS O-ASCs

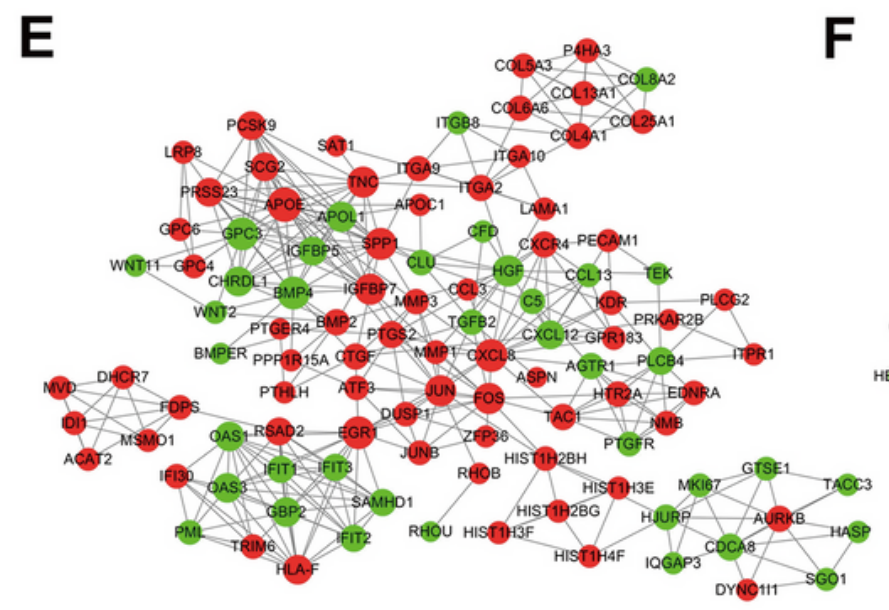

O-ASCs VS Y-ASCs
$\mathbf{F}$

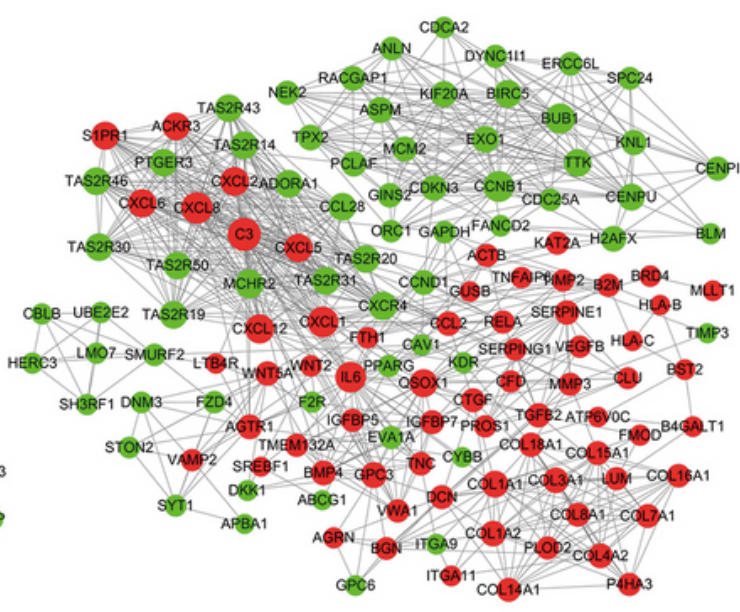

D-ASCs VS O-ASCs

\section{Figure 3}

Bioinformatics analyses of the differentially expressed mRNAs. (A, B, E) GO, KEGG pathway and PPI network analyses of the differentially expressed mRNAs in O-ASCs vs Y-ASCs. (C, D, F) GO, KEGG pathway and PPI network analyses of the differentially expressed mRNAs in D-ASCs vs O-ASCs. 
A

QPCR

B
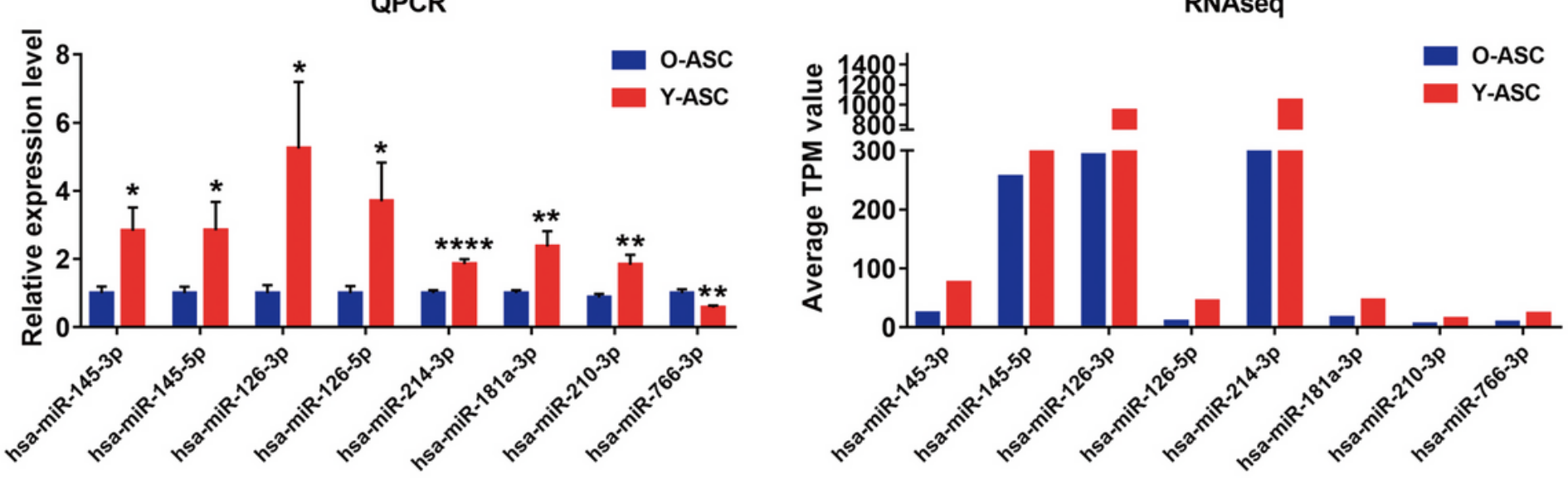

C

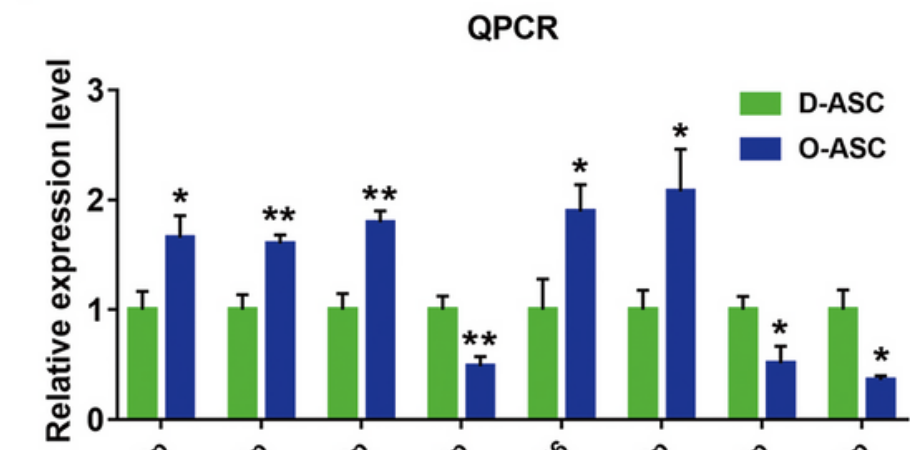

D
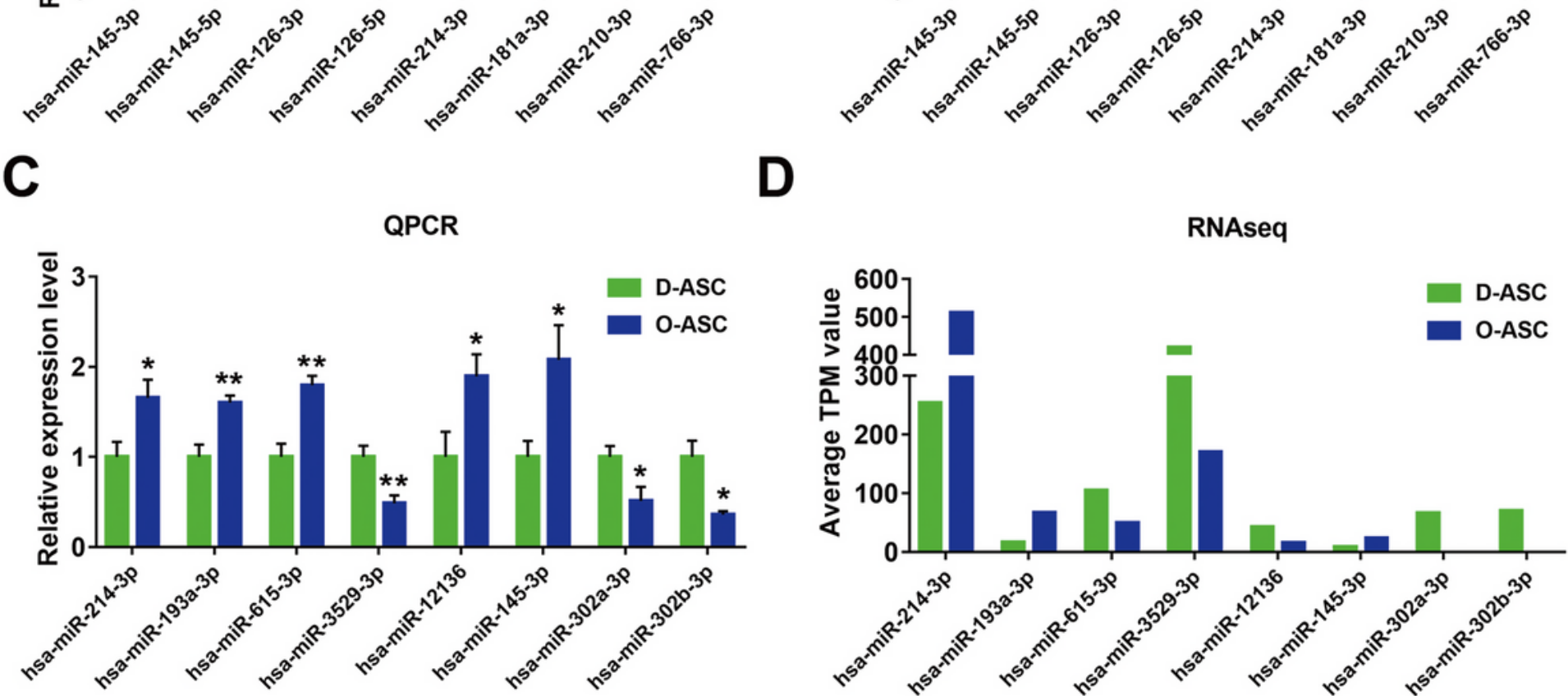

$\mathbf{E}$

QPCR
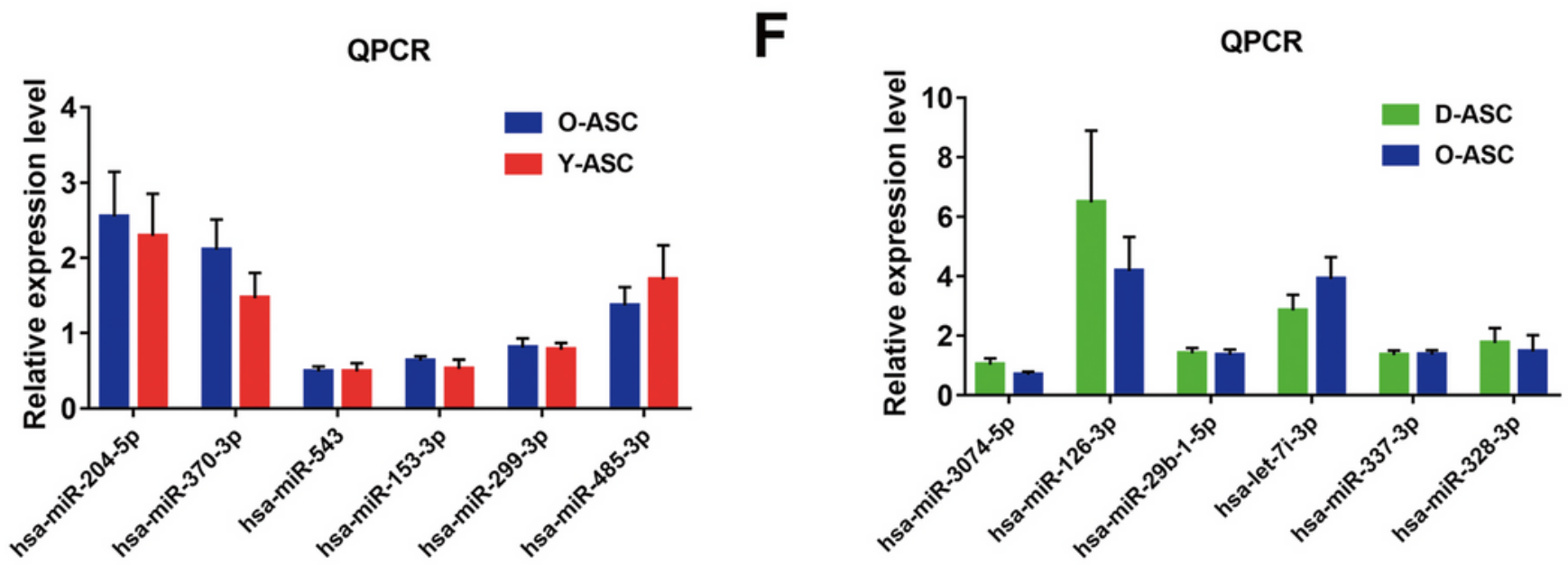

Figure 4

Real-time PCR analysis of the differentially expressed miRNAs. (A) Real-time PCR validation of the differentially expressed miRNAs comparing O-ASCs with Y-ASCs. N=9. (B) RNA-seq profiles of the selected miRNAs in (A). (C) Real-time PCR validation of the differentially expressed miRNAs comparing DASCs with O-ASCs. N=5. (D) RNA-seq profiles of the selected miRNAs in (C). miRNAs with no significant difference by PCR analysis were shown in (E-F). $N=5 .{ }^{*} p<0.05,{ }^{*} p<0.01, * \star \star p<0.001,{ }^{* \star *} p<0.0001$. 
A

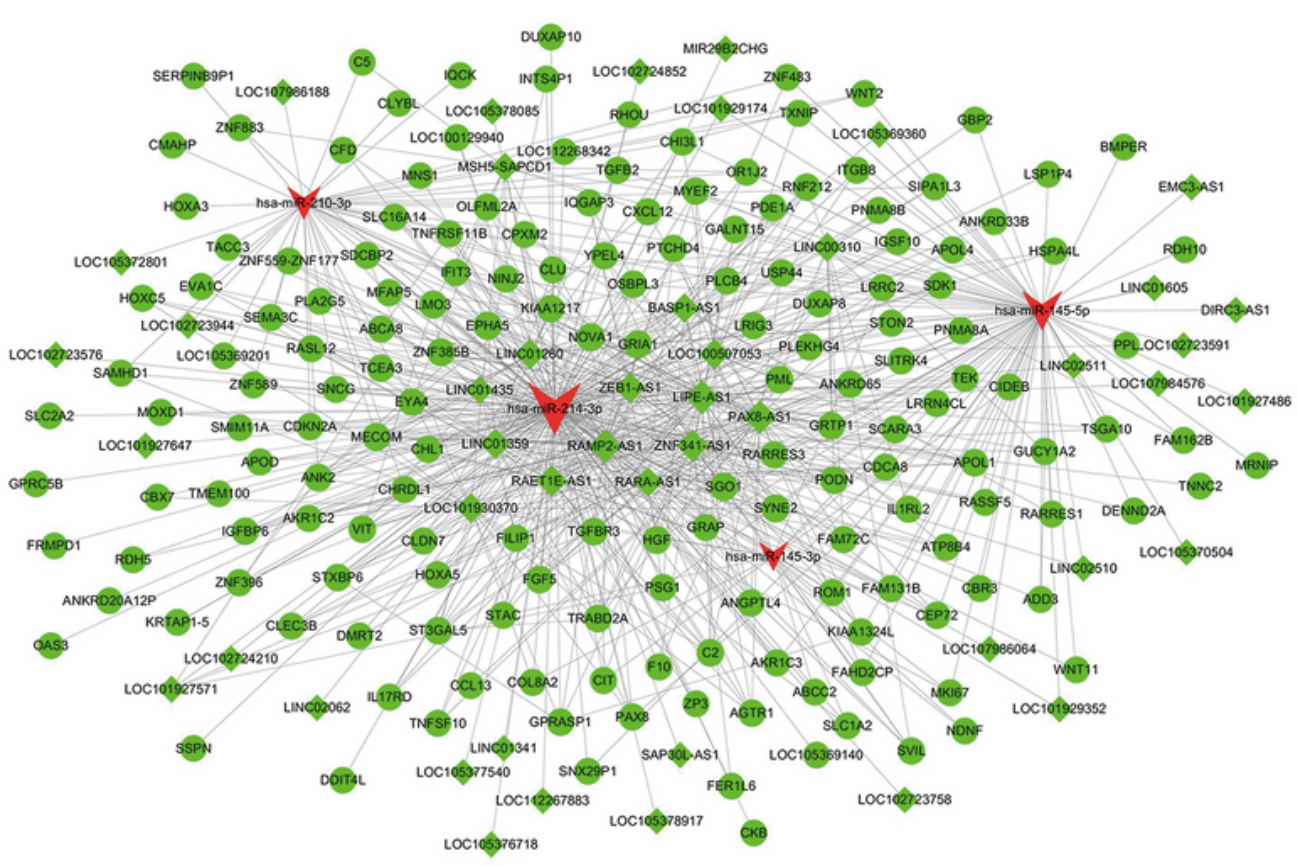

B

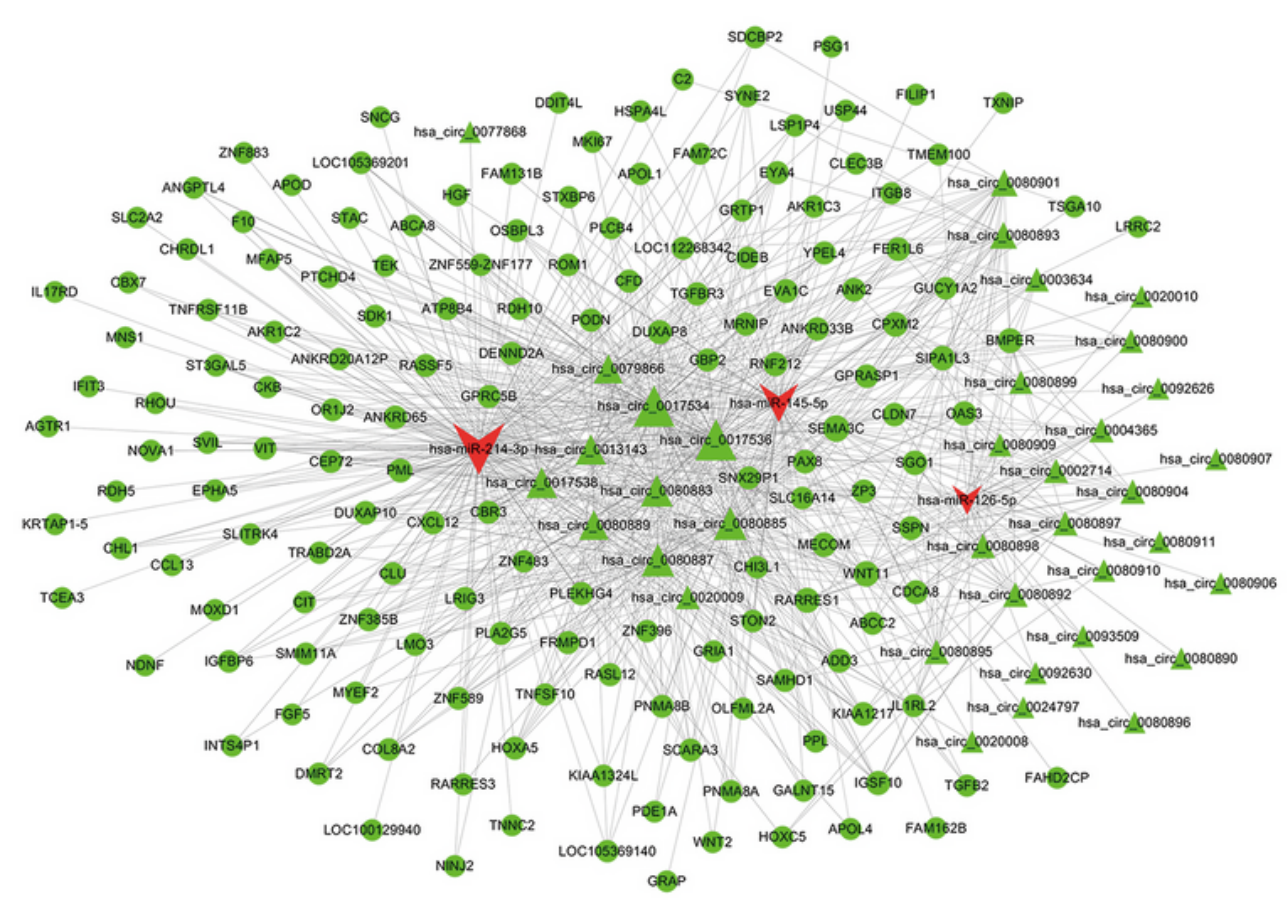

CircRNA-miRNA-mRNA network O-ASCs VS Y-ASCs

\section{Figure 5}

CeRNA network construction in O-ASCs vs Y-ASCs. IncRNAs (A) and cirRNAs (B) - miRNAs - mRNAs networks were constructed based on the differentially expressed IncRNAs, circRNAs, mRNAs and validated miRNAs. 


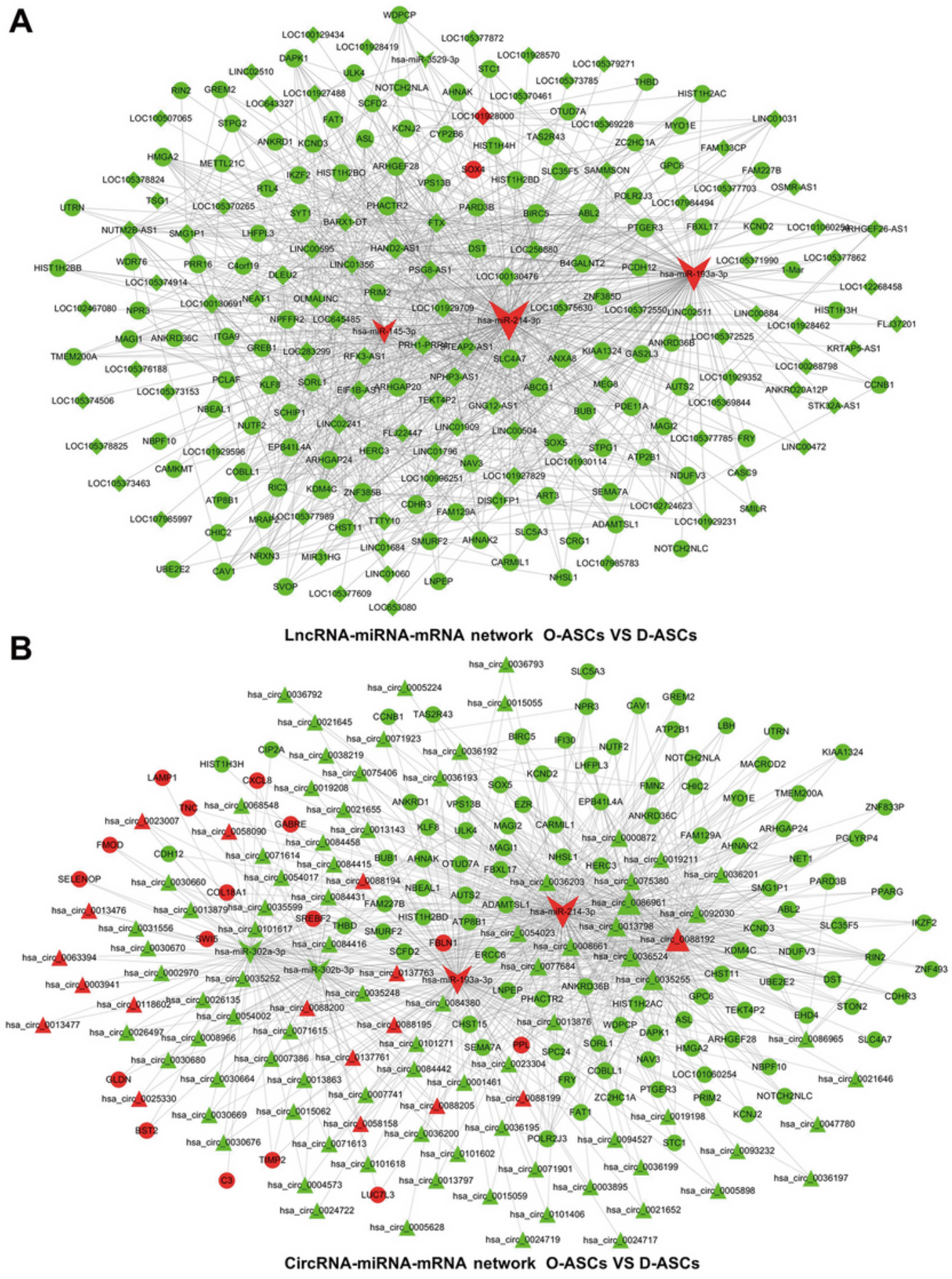

\section{Figure 6}

CeRNA network construction in D-ASCs vs O-ASCs. IncRNAs (A) and cirRNAs (B) - miRNAs - mRNAs networks were constructed based on the differentially expressed IncRNAs, circRNAs, mRNAs and validated miRNAs. 
A

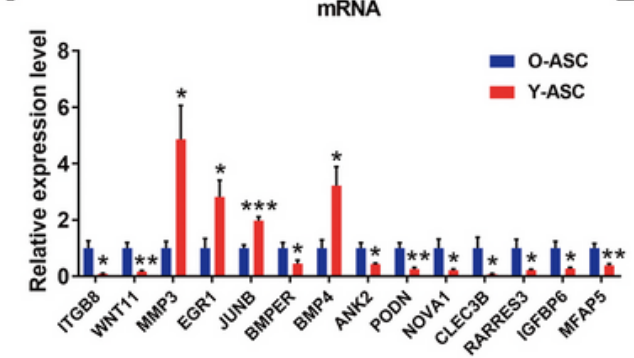

C

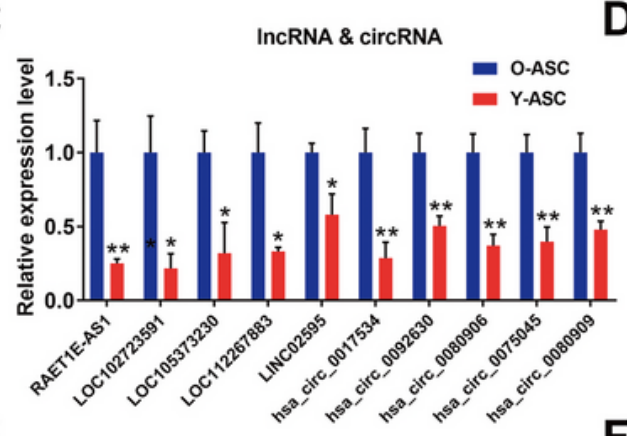

E

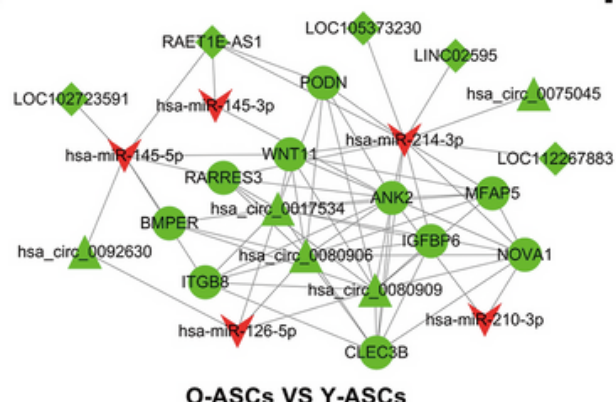

B

$\mathbf{F}$

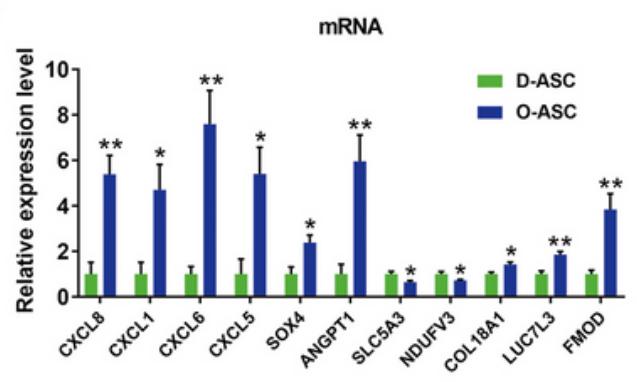

D
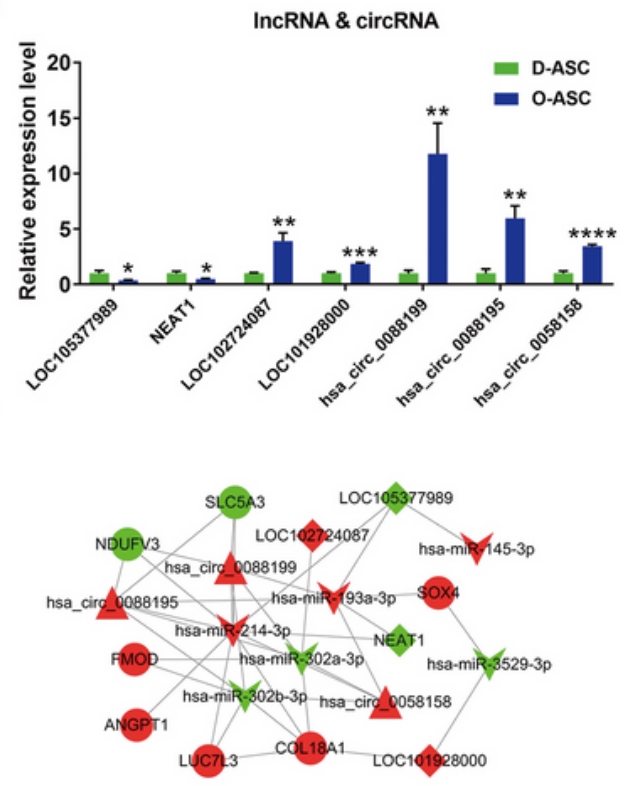

D-ASCs Vs O-ASCs

I

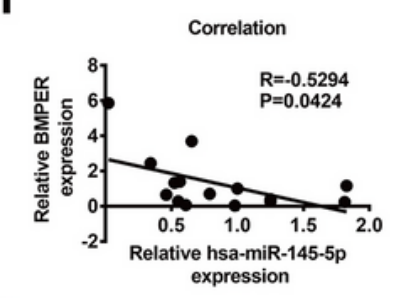

J

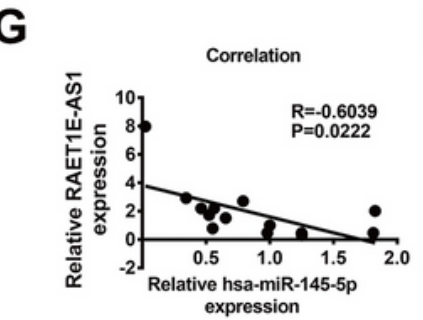

H
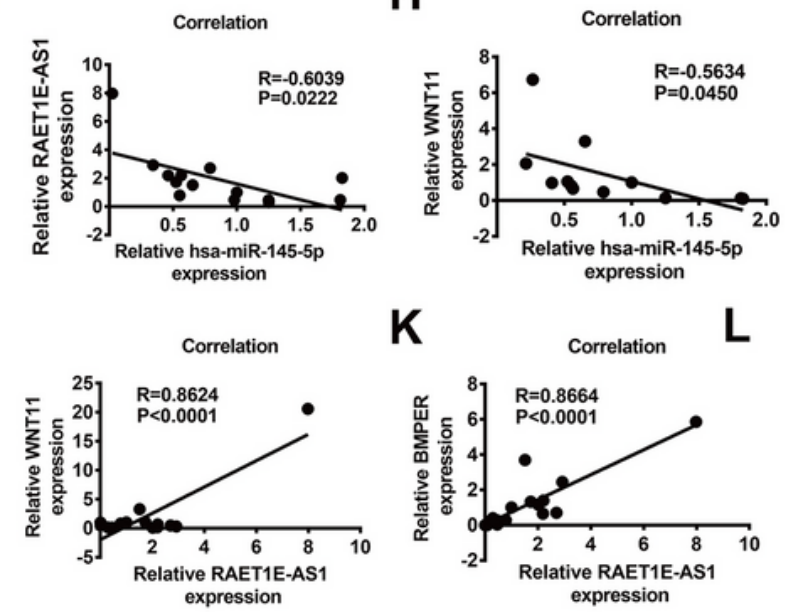

K
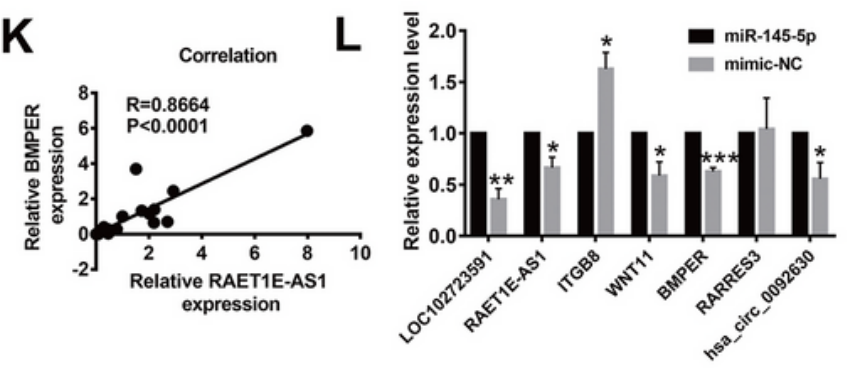

\section{Figure 7}

Real-time PCR analysis of RNAs in above PPI and ceRNA networks, and construction of ceRNA subnetworks. (A, B) Real-time PCR validation of the selected mRNAs comparing Y-ASCs with O-ASCs and DASCs with O-ASCs. N=5. (C, D) Real-time PCR validation of the selected IncRNAs and circRNAs comparing Y-ASCs with O-ASCs and D-ASCs with O-ASCs. N=5. (E, F) Construction of ceRNA sub-networks based on the above PCR results. (G-I) Correlation analysis of the expression level of miR-145-5p with that of 
RAET1E-AS1, WNT11 and BMPER. N=13-15. (J, K) Correlation analysis of the expression level of RAET1EAS1 with that of WNT11 and BMPER. N=15, 17. (L) Real-time PCR analysis of the expressional changes of RNAs in the ceRNA sub-network after miR-145-5p overexpression in ASCs. ${ }^{*} p<0.05,{ }^{\star \star} p<0.01,{ }^{* \star} p<$ $0.001, * \star \star \star p<0.0001$.

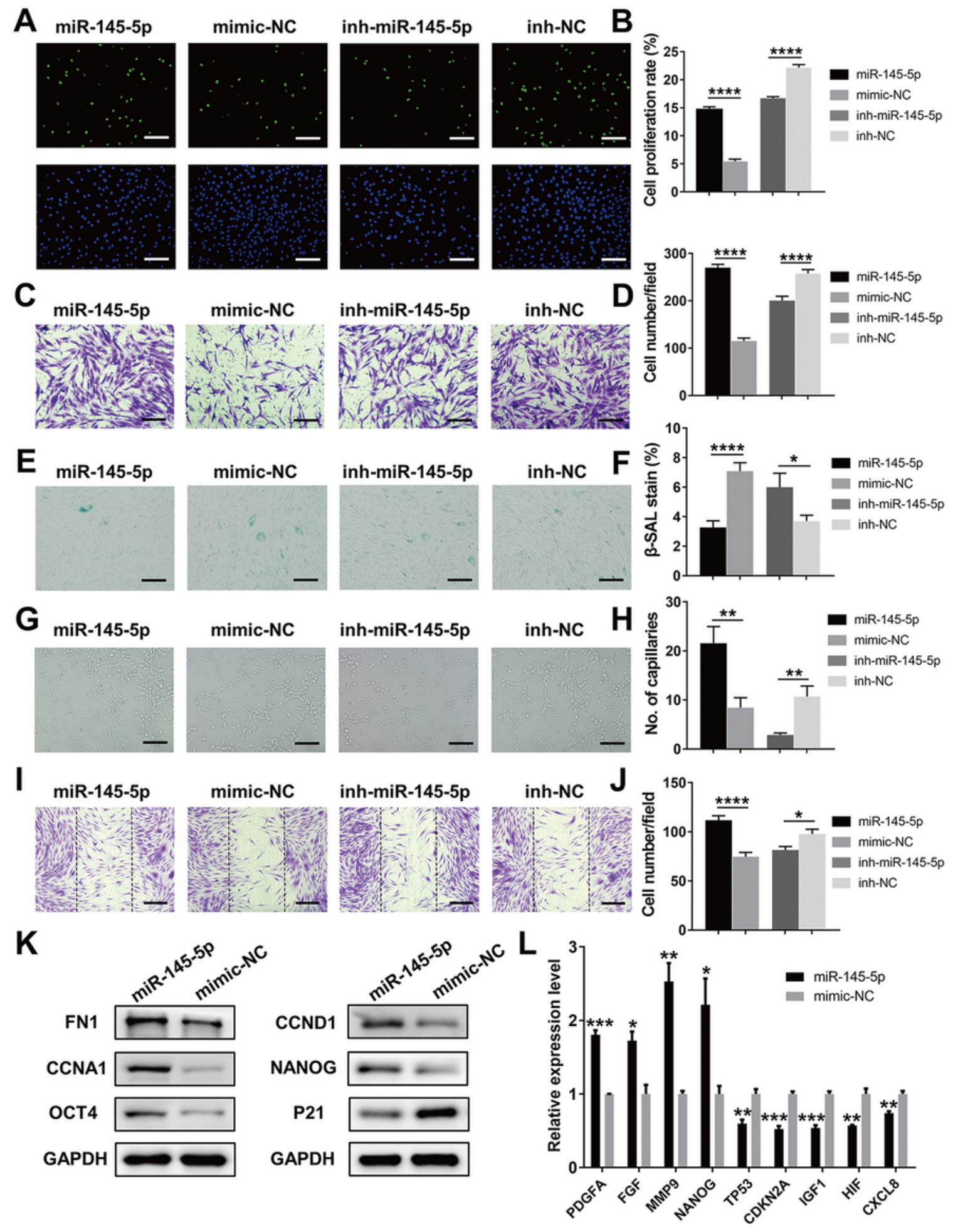

Figure 8 
Overexpression of miR-145-5p rejuvenated old ASCs and enhanced their function. (A) The proliferative rate of ASCs treated with miR-145-5p, mimic-NC, inh- miR-145-5p and inh-NC were measured by EdU assay. Scar bar: $50 \mu \mathrm{m}$. (C) Images of migrated ASCs given above treatments. Scar bar: $50 \mu \mathrm{m}$. (E) $\beta$-gal Staining assay analysis of the senescent rate of ASCs given above treatments. Scar bar: $50 \mu \mathrm{m} .(\mathrm{G})$ Images of tube formations in HUVECs treated with conditional media of ASCs given above treatments. Scar bar: $50 \mu \mathrm{m}$. (I) Images of migrated fibroblasts given above treatments. Scar bar: $100 \mu \mathrm{m}$. (B, D, F, H, J) qualified data shown in (A, C, E, G, I) separately. (K) Western blot analysis of the proteins levels in ASCs after miR-145-5p overexpression. (L) Real-time PCR analysis of the genes expressions in ASCs after miR145-5p overexpression. ${ }^{*} p<0.05,{ }^{\star *} p<0.01,{ }^{* \star *} p<0.001,{ }^{\star \star \star *} p<0.0001$. 


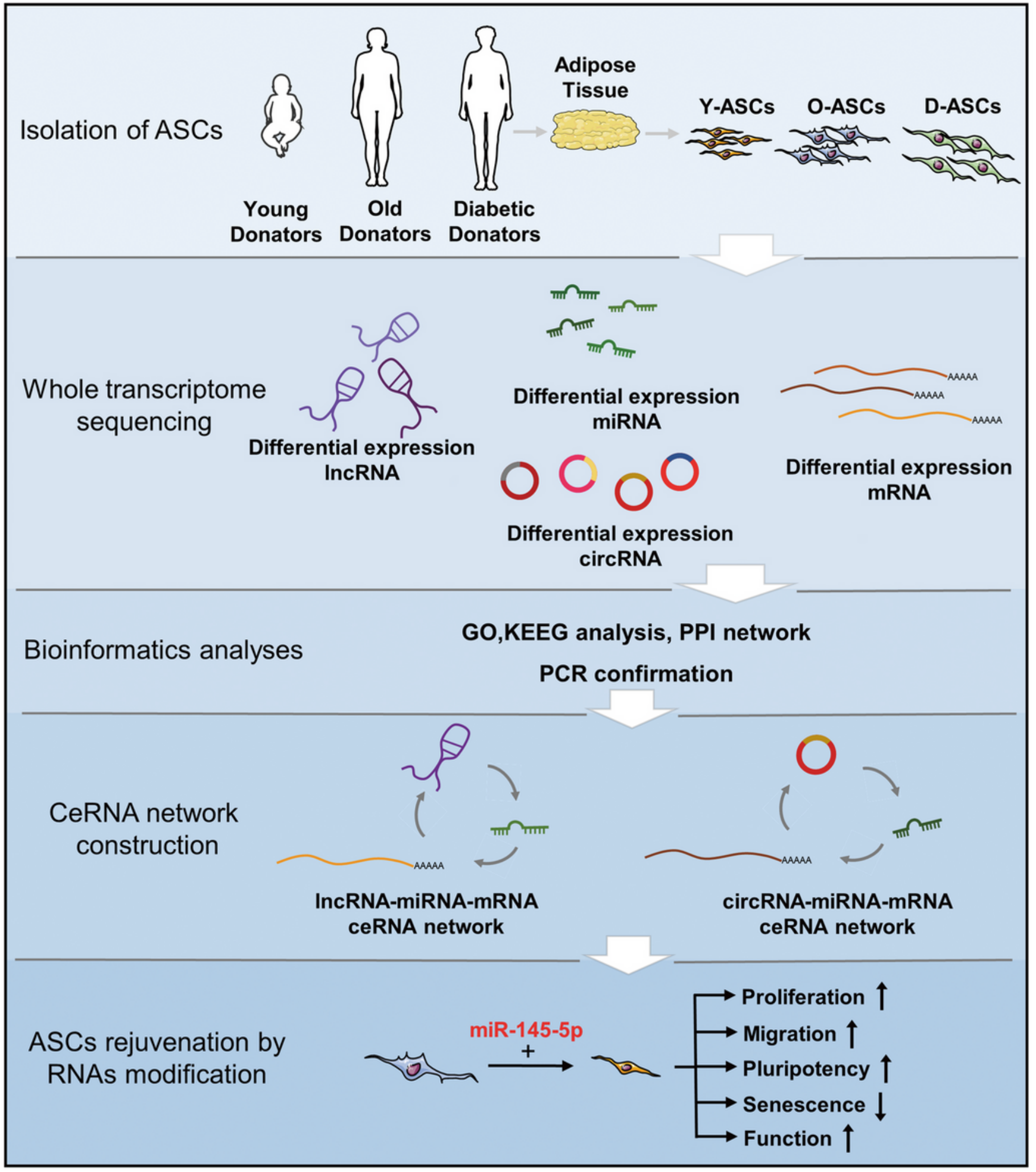

Figure 9

The flow diagram of this study.

\section{Supplementary Files}

This is a list of supplementary files associated with this preprint. Click to download. 
- Additionalfile1.TableS15.docx

- Additionalfile2.FigureS1.pdf

- Additionalfile3.DataS1.xlsx

- Additionalfile4.DataS2.xlsx

- Additionalfile5.DataS3.xlsx

- Additionalfile6.DataS4.xls

- Additionalfile7.DataS5.xIsx

- Additionalfile8.DataS6.xls

- Additionalfile9.DataS7.xls

- Additionalfile10.DataS8.xls

- Additionalfile11.DataS9.xlsx

- Additionalfile12.DataS10.xIsx

- Additionalfile13.DataS11.xlsx

- Additionalfile14.DataS12.xlsx

- Additionalfile15.DataS13.xIsx

- Additionalfile16.DataS14.xlsx

- Additionalfile17.DataS15.xlsx

- Additionalfile18.DataS16.xIsx 